\title{
NGC 1980 Is Not a Foreground Population of Orion: Spectroscopic Survey of Young Stars with Low Extinction in Orion A
}

\author{
Min Fang ${ }^{1}$, Jinyoung Serena Kim ${ }^{1}$, Ilaria Pascucci ${ }^{2}$, Dániel Apai ${ }^{1,2}$, Lan Zhang ${ }^{3,4}$, Aurora Sicilia-Aguilar ${ }^{5}$, \\ Miguel Alonso-Martínez ${ }^{6}$, Carlos Eiroa ${ }^{6}$, and Hongchi Wang $^{7}$ \\ ${ }^{1}$ Department of Astronomy, University of Arizona, 933 North Cherry Avenue, Tucson, AZ 85721, USA \\ ${ }^{2}$ Department of Planetary Sciences, University of Arizona, 1629 East University Boulevard, Tucson, AZ 85721, USA \\ ${ }^{3}$ Key Lab of Optical Astronomy, National Astronomical Observatories, CAS, 20A Datun Road, Chaoyang District, 100012 Beijing, China \\ ${ }^{4}$ CAS South America Center for Astronomy, Camino El observatorio \#1515, Las Condes, Santiago, Chile \\ ${ }^{5}$ SUPA, School of Physics and Astronomy, University of St. Andrews, North Haugh, St. Andrews KY16 9SS, UK \\ ${ }^{6}$ Departamento de Fisica Teorica, Facultad de Ciencias, Universidad Autonoma de Madrid, E-28049 Cantoblanco, Madrid, Spain \\ ${ }^{7}$ Purple Mountain Observatory and Key Laboratory of Radio Astronomy, Chinese Academy of Sciences, 2 West Beijing Road, 210008 Nanjing, China \\ Received 2016 September 19; revised 2017 February 28; accepted 2017 March 1; published 2017 March 30
}

\begin{abstract}
We perform a spectroscopic survey of the foreground population in Orion A with MMT/Hectospec. We use these data, along with archival spectroscopic data and photometric data, to derive spectral types, extinction values, and masses for 691 stars. Using the Spitzer Space Telescope data, we characterize the disk properties of these sources. We identify 37 new transition disk (TD) objects, 1 globally depleted disk candidate, and 7 probable young debris disks. We discover an object with a mass of less than $0.018-0.030 M_{\odot}$, which harbors a flaring disk. Using the $\mathrm{H} \alpha$ emission line, we characterize the accretion activity of the sources with disks, and confirm that the fraction of accreting TDs is lower than that of optically thick disks $(46 \% \pm 7 \%$ versus $73 \% \pm 9 \%$, respectively). Using kinematic data from the Sloan Digital Sky Survey and APOGEE INfrared Spectroscopy of the Young Nebulous Clusters program (IN-SYNC), we confirm that the foreground population shows similar kinematics to their local molecular clouds and other young stars in the same regions. Using the isochronal ages, we find that the foreground population has a median age of around 1-2 Myr, which is similar to that of other young stars in Orion A. Therefore, our results argue against the presence of a large and old foreground cluster in front of Orion A.
\end{abstract}

Key words: accretion, accretion disks - protoplanetary disks - stars: pre-main sequence

Supporting material: machine-readable tables

\section{Introduction}

The Orion complex is the most active star-forming region in the solar neighborhood. The age range of $(<1-10 \mathrm{Myr})$, the diversity of local environments, and the distance $(\sim 414 \mathrm{pc}$, Menten et al. 2007) of this complex make it an ideal laboratory to study challenging questions in star formation, e.g., starformation history, initial mass function, and disk evolution (see, e.g., Hillenbrand 1997; Hernández et al. 2007a, 2007b; Fang et al. 2009, 2013a; Lee \& Chen 2009; Hernández et al. 2010; Hsu et al. 2012, 2013; Hernández et al. 2014).

The entire Orion complex shows evidence for multiple starformation episodes, and the current, most active star formation is located in Orion A and B. Orion A, located in the southern part, consists of the most massive cluster in this region, the Orion nebula cluster, and several medium-size clusters, e.g., OMC 2, OMC 3, and Lynds 1641 (L1641). In L1641, about half of the young stellar objects (YSOs) are formed in isolation and others are formed in small aggregates (Fang et al. 2013a). Orion B is in the northern part of Orion and is comprised of four clusters NGC 2023, 2024, 2068, and 2071. There is also a large population of off-cloud stellar groups aged 3-30 Myr, including the Orion OB1a and 1B associations (Briceño et al. 2005, 2007). The understanding of star formation in the Orion complex was further complicated by the discovery of a foreground population aged 4-5 Myr to Orion A (Alves \& Bouy 2012; Bouy et al. 2014). This presumably older population is centered on NGC 1980 ( $\iota$ Ori), and extends northward to the Orion nebula cluster and NGC 1981, and eastward to L1641, thus contaminating the young populations in these regions.

In Alves \& Bouy (2012), the foreground population was proposed by searching for the stars with low or no extinction in the field of Orion A. The surface densities of this population show a well-defined peak coinciding with NGC 1980, and several less distinct peaks around other star-forming regions in Orion A (Alves \& Bouy 2012; Bouy et al. 2014). In the $J-H$ versus $H-K_{\text {s }}$ color-color diagram, the stars in the population show near-infrared colors consistent with B- to M-type stars (Alves \& Bouy 2012). Therefore, Alves \& Bouy (2012) propose that the NGC 1980 cluster is a foreground population seen in projection against the Orion A cloud. Alves \& Bouy (2012) estimate the age of the NGC 1980 cluster on the basis of the median spectral energy distribution (SED) and the age of a massive star, $\iota$ Ori, which is $4-5$ Myr. However, previous studies are all based only on photometric data and lack spectroscopic follow-up. In this work, we provide a spectroscopic study of the putative foreground population, and address the issue of its age and other properties. Our results suggest that NGC 1980 is actually associated with the Orion A cloud and has an age similar to other star-forming regions in Orion A. We organized the paper as follows. In Section 2, we will describe our observations and data reduction. In Section 3, we will delineate our data analysis. We will present our results in Section 4, followed by a discussion in Section 5, and we will summarize our results in Section 6. 


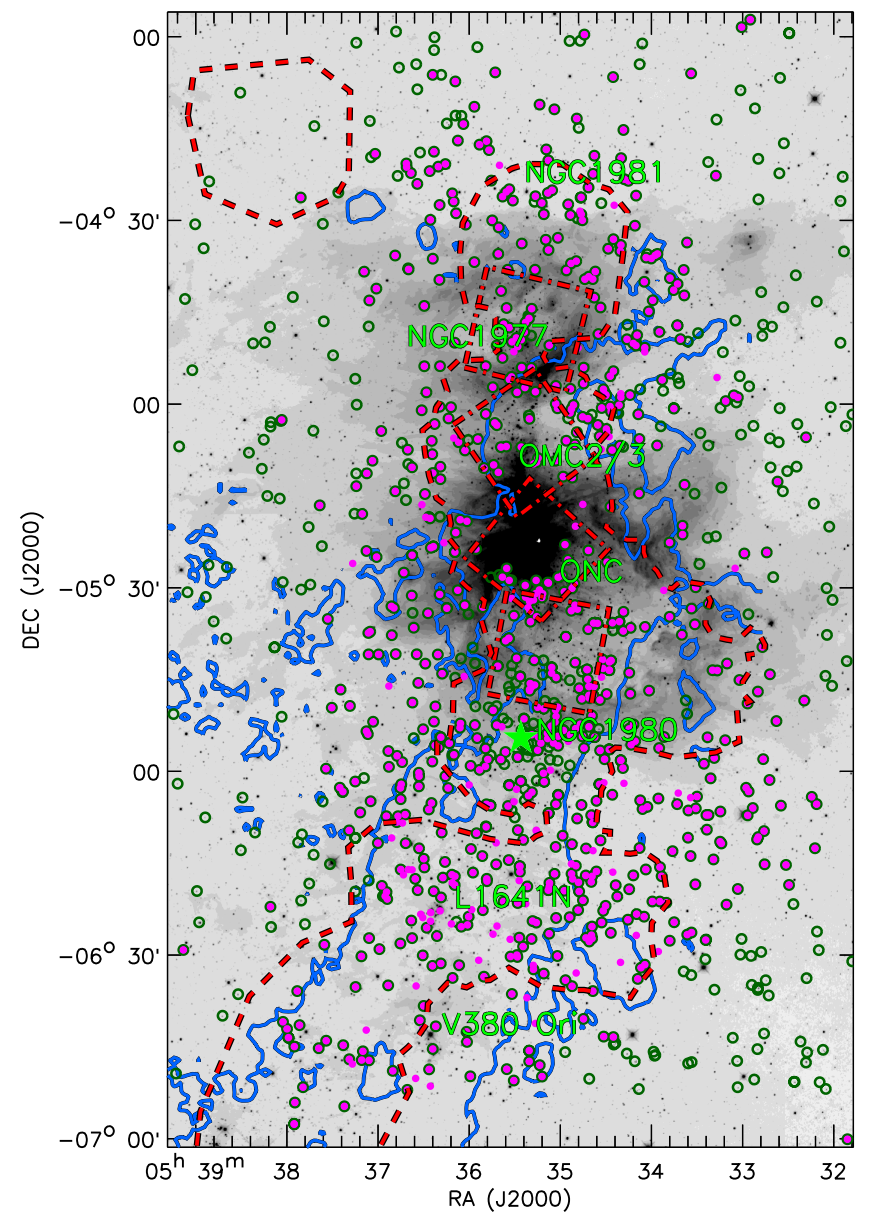

Figure 1. WISE 3.4 micron image of Orion A. The circles (open dark green and magenta filled) show all the stars with probabilities of more than $99 \%$ of being members of NGC 1980 and brighter than 19 mag in $r$ band. The magenta filled circles mark the stars with spectroscopic data. The green filled star symbol marks the position of the massive star $\iota$ Ori. The blue solid-line contours are the ${ }^{13} \mathrm{CO}$ integrated intensity from Bally et al. (1987) with a contour level of $5 \mathrm{~K} \mathrm{~km} \mathrm{~s}^{-1}(5 \sigma)$. The red dashed lines enclose the field of view (FOV) of the $X M M$ observations, and the red dashed-dotted line boxes are for the FOVs of the Chandra observations.

\section{Observations and Data Reduction}

\subsection{Spectroscopic Observations}

\subsubsection{Target Selection}

Bouy et al. (2014) lists 1895 sources with the probability that more than $99 \%$ are members of NGC 1980. These stars are distributed over several tens of square degrees with the stellar surface densities peaking around the Orion molecular cloud. Our targets for the spectroscopic study of NGC 1980 are selected from Bouy et al. (2014), and are limited to the vicinity of the Orion cloud. In Figure 1, we show the regions we studied. In this area, there are 1275 sources with with the probability of more than $99 \%$ being members of NGC 1980. Hereafter, these sources are considered to be high-confidence cluster members. We search for the available spectral types for these high-confidence cluster members in the literature (Hillenbrand 1997; Rebull et al. 2000; Rebull 2001; Fang et al. 2009, 2013a; Hillenbrand et al. 2013; Hsu et al. 2012), and in our unpublished data from our previously spectroscopic survey with MMT/Hectospec and WHT/AF2 (M. Fang et al. 2017 , in preparation). We obtain spectral types for 330 sources

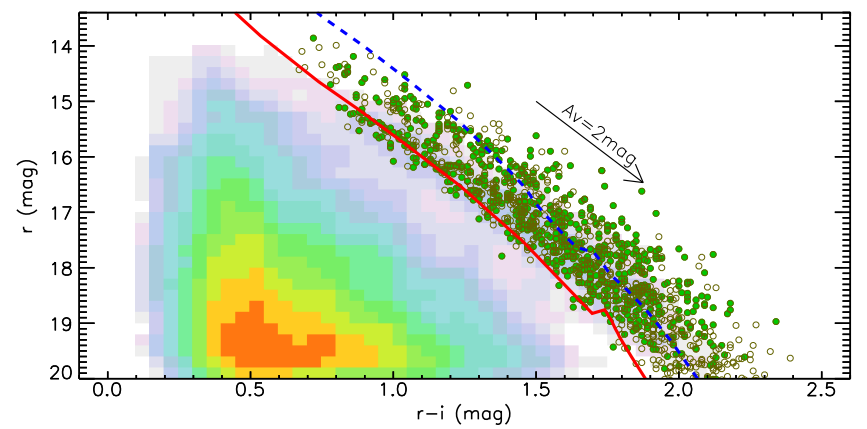

Figure 2. Hess $r-i$ vs. $r$ color-magnitude diagram for all the stars in the field of Orion from Bouy et al. (2014). The circles (open and filled) show all the high-confidence members of NGC 1980 in the field shown in Figure 1, and the filled circles are for those with spectroscopic data. The solid line shows the $3 \mathrm{Myr}$ isochrone at an distance of $414 \mathrm{pc}$ calculated for the PMS evolutionary models of Baraffe et al. (2015), using the BT-Settl model atmospheres (Allard et al. 2011), with the solar abundances from Asplund et al. (2009). The dashed line is similar to the solid line, but for a $4.5 \mathrm{Myr}$ isochrone at a distance of $210 \mathrm{pc}$. The arrow shows the extinction vector with visual extinction of $2 \mathrm{mag}$.

from published literature, and for 172 sources from our unpublished data sets. Due to limited observing time, we only selected a subsample of high-confidence cluster members for spectroscopic observations with MMT/Hectospec. In Figure 2, we show the $r$ versus $r-i$ color-magnitude diagram for highconfidence cluster members, as well for sources with known spectral types and stars we selected for the MMT/Hectospec observations. We calculate the $3 \mathrm{Myr}$ isochrone in the $r-i$ versus $r$ color-magnitude diagram for the PMS stellar evolutionary models of Baraffe et al. (2015), using the BT-Settl model atmospheres (Allard et al. 2011), with solar abundances from Asplund et al. (2009). ${ }^{8}$ As a comparison, we show the isochrone in Figure 2, assuming a distance 414 pc (Menten et al. 2007). Most of our targets are located above this isochrone, indicating that they are younger than $3 \mathrm{Myr}$. However, the ages of our targets could be older, if they lie much closer than Orion A. If we assume an age of $\sim 4-5 \mathrm{Myr}$ for our targets as suggested in Alves \& Bouy (2012), they should be located at $\sim 210 \mathrm{pc}$ to match their position in the color-magnitude diagram (see Figure 2). However, Alves \& Bouy (2012) argue that the distances of these sources would not be substantially different from the ones in Orion, and might be around $400 \mathrm{pc}$, since they show similar kinematics to the Orion A molecular cloud. Therefore, the ages and the distance for these sources assumed in Alves \& Bouy (2012) are inconsistent.

\subsubsection{Spectroscopic Observations and Data Reduction}

We performed a low-resolution spectroscopic survey of the stellar population in NGC 1980 with the Hectospec multiobject spectrograph, capable of taking a maximum of 300 spectra simultaneously. We used the 270 groove $\mathrm{mm}^{-1}$ grating and obtained spectra in the 3700-9000 $\AA$ range with a spectral resolution of $\sim 5 \AA$. The targets are selected from Bouy et al. (2014), and have probabilities $\geqslant 99 \%$ as members of NGC 1980. Our $\sim 300$ sources are distributed at four pointings. The data were taken in 2016 February. Table 1 lists the observational logs.

\footnotetext{
8 A discussion on the BT-Settl models with two types of solar abundances (Asplund et al. 2009; Caffau et al. 2011) can be found in Section 3.3.
} 
Table 1

Hectospec Observation Logs

\begin{tabular}{|c|c|c|c|c|c|}
\hline \multirow[b]{2}{*}{ Config. } & \multirow[b]{2}{*}{ Obs-date (UT) } & \multirow{2}{*}{$\begin{array}{c}\text { R.A. } \\
\text { (J2000) } \\
\text { (h:m:s) }\end{array}$} & \multirow{2}{*}{$\begin{array}{c}\text { Decl. } \\
(\mathrm{J} 2000) \\
(\mathrm{d}: \mathrm{m}: \mathrm{s})\end{array}$} & \multicolumn{2}{|c|}{ Exposure } \\
\hline & & & & $\begin{array}{c}\text { Obj } \\
\text { (minutes) }\end{array}$ & $\begin{array}{c}\text { sky } \\
\text { (minutes) }\end{array}$ \\
\hline 1 & 2016 Feb 4 & $+05: 33: 54.6$ & $-06: 01: 19$ & $3 \times 15$ & $1 \times 15$ \\
\hline 2 & 2016 Feb 4 & $+05: 35: 51.5$ & $-05: 48: 52$ & $3 \times 15$ & $1 \times 15$ \\
\hline 3 & 2016 Feb 6 & $+05: 35: 15.9$ & $-04: 32: 58$ & $3 \times 15$ & $1 \times 15$ \\
\hline 4 & 2016 Feb 8 & $+05: 35: 32.2$ & $-05: 22: 39$ & $3 \times 15$ & $1 \times 15$ \\
\hline
\end{tabular}

We use standard IRAF routines to reduce the Hectospec data according to standard procedures. We perform flat-field correction and extract the spectra using dome flats with the IRAF task dofibers in the package specred. The wavelength solution for Hectospec is obtained with HeNeAr comparison spectra, using the IRAF task identify and reidentify in the package specred. We calibrate the spectra with a wavelength solution constructed using the IRAF task dispcor under the package specred. For each pointing, we took three exposures for the science targets, and one exposure for the sky by shifting the telescope by $\sim 5$ arcsec. We extracted the spectra for each exposure. Finally, we obtained the spectra for each target and the corresponding sky spectra close to this target. We subtracted the sky from the spectra of each target, and combined the sky-subtracted spectra into one final spectrum.

\subsection{Photometric Data}

The photometric data used in this work are mainly from Bouy et al. (2014), in which they combine the photometric data from the Sloan Digital Sky Survey (SDSS, York et al. 2000), CTIO/DECam, the Two-Micron All Sky Survey (2MASS, Skrutskie et al. 2006), the UKIRT Infrared Deep Sky Survey (UKIDSS, Lawrence et al. 2007), and the AAVSO Photometric All-Sky Survey (Henden et al. 2016), and present a catalog with photometry in the grizYJHKs bands. The photometric data from CTIO/DECam are in griz $Y$ bands, and have been calibrated in flux by cross-matching the common stars in the SDSS DR9 catalog in griz bands, and in the UKIDSS catalog in $Y$ band (see Bouy et al. 2014). We complement them with the Spitzer data from Megeath et al. (2012), and Wide-field Infrared Survey Explorer (WISE, Wright et al. 2010). These infrared data are used to characterize the circumstellar disks.

\subsection{X-Ray Data}

Low-mass young stars usually present strong magnetic activity, and show two to three orders of magnitude brighter $\mathrm{X}$-ray emission than the field population (Feigelson \& Montmerle 1999; Feigelson et al. 2007). Thus X-ray data can be used to distinguish young stars from field stars in starforming regions, particularly diskless (Class III) stellar population (Feigelson et al. 2007). Part of the region studied here has been observed by the X-ray space telescopes $X M M$ Newton and Chandra. The sky coverage of the XMM (Proposal IDs: 004956, 008994, 009300, 011259, 011266, 013453, 040657, 050356, 060590, and 069020) and Chandra (Proposal IDs: 01200704, 03200289, and 04200331) observations are shown in Figure 1. The X-ray data will be used to characterize the youth of our sources.

\section{Data Analysis \\ 3.1. Spectral Classification}

The spectral types of young stars are usually obtained by classifying their observed spectra. This is typically done by building a relation between the strength of spectral features and the spectral types of dwarf stars, and applying them to the young stars (Hillenbrand 1997; Hernández et al. 2004). Uncertainties on the spectral classification can be several subclasses depending on the spectral features and/or ranges used for the determination. One typical example is TW Hya, of which the spectral type ranges from K6 to M2.5 (de la Reza et al. 1989; Torres et al. 2006; Vacca \& Sandell 2011; Pecaut \& Mamajek 2013; Herczeg \& Hillenbrand 2014). Pecaut \& Mamajek (2013) recently studied a sample of young stars in nearby groups: the $\eta$ Cha cluster, the TW Hydra Association, the $\beta$ Pic Moving Group, and the Tucana-Horologium Association. We found that the spectral types in Pecaut \& Mamajek (2013) for the same stars are typically one subclass different from those in the literature. Though it is not clear which classification results are more accurate, we decide to classify our stars based on the same criteria as those in Pecaut \& Mamajek (2013).

We select X-Shooter spectra for 85 young stars from the ESO Phase 3 spectral data archive. Among them, 27 young stars in the $\eta$ Cha cluster and the TW Hydra Association have been spectrally classified by Pecaut \& Mamajek (2013). We use these spectra as the templates to classify the other sources with X-Shooter spectra. Using these X-Shooter spectra, we construct new relations between the strengths of spectral features and spectral types for young stars in the range of K2M9.5 (see the detailed description in Appendix A). The new relations should be consistent with the classification criteria from Pecaut \& Mamajek (2013). Thus, the conversion from spectral types to effective temperatures, intrinsic colors, and bolometric corrections for young populations in Pecaut \& Mamajek (2013) can be used for other young stars with spectral types classified based on our relations. In this work, we use these relations to classify the spectra from Hectospec. In Figure 3, we compare the spectral types from our relations and from the SPTCLASS code (Hernández et al. 2004). It can be noted that both spectral types are consistent with each other given the uncertainty within the range of M0-M6. When the spectral types are later than M6 or earlier than M0, our relations give about one subclass earlier spectral type than SPTCLASS.

\subsection{YSO Selection Criteria}

The targets in this work are obtained from a sample of candidate PMS stars selected from different sets of colormagnitude diagrams by Bouy et al. (2014). Some of them could be interlopers from main-sequence (MS) stars or giants. In this 


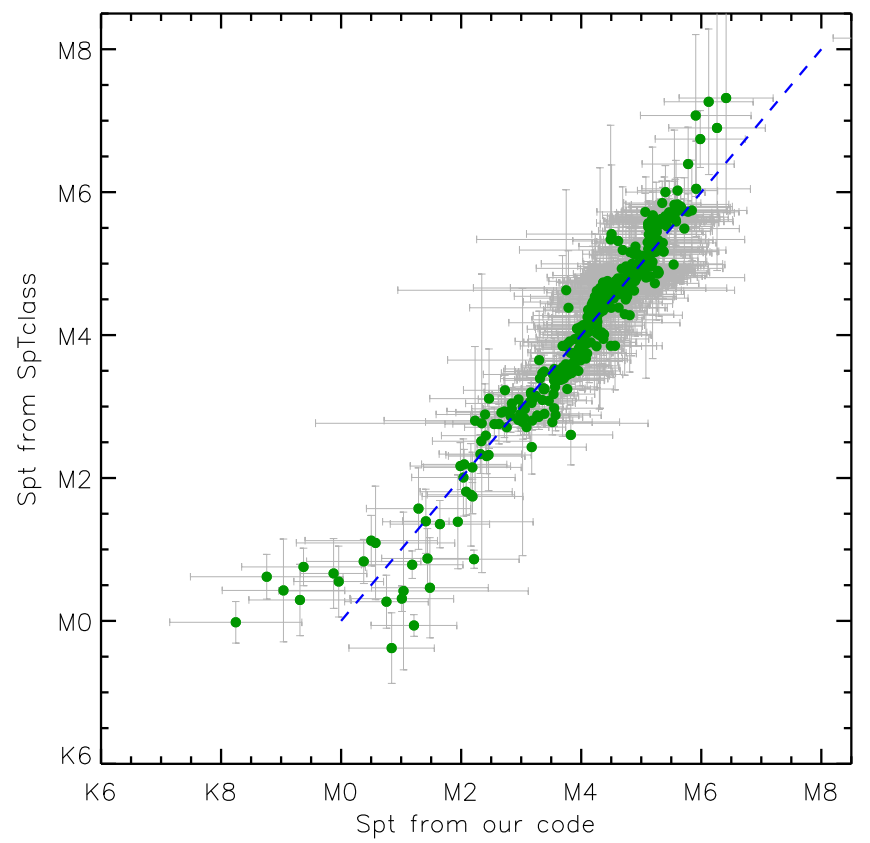

Figure 3. Comparison of spectral types from our code and from the SPTCLASS code (Hernández et al. 2004). The uncertainty for each spectral type is indicated. The dashed line marks where the two spectral types are equal in the M-type range.

section, we will clarify our criteria to remove the contaminators in our sample.

\subsubsection{Spectral Features}

The youth of the stars can be characterized using several indicators. The typical one is the Li I absorption line at $6708 \AA$. In Figure 4 , we show the examples of $\mathrm{Li}$ I $\lambda 6708 \AA$ detections for threee sources with MMT/Hectospec. Among the 752 sources in our sample, there are 444 sources for which we have obtained spectra. Among them, 295 stars show Li I $\lambda 6708 \AA$ absorption, 11 do not show clear Li I $\lambda 6708 \AA$ absorption, and others have spectra that are too noisy for Li I $\lambda 6708 \AA$ to be identified. An additional 152 stars are found to show Li I absorption in Hsu et al. (2012). In total, a group of 447 stars in our sample are identified as young stars based on the Li I $\lambda 6708 \AA$ absorption. In Table 6, we list the equivalent width (EW) of the Li I $\lambda 6708 \AA$ absorption for those stars. The typical uncertainty of the Li I $\lambda 6708 \AA$ EWs is around $0.1-0.2 \AA$.

The strength of the NaI doublets at 8183 and $8195 \AA$ has proved to be a good indicator for stellar surface gravitiy for M-type stars (Lawson et al. 2009). MS stars usually show strong Na I doublets, and giants present very weak ones. The PMS stars show the strengths of the NaI doublets between the mainsequence stars and giants. In this work, we will use the Index (Na I) as a second indicator to select the PMS stars. We calculate the strength of the $\mathrm{NaI}$ doublets at 8183 and $8195 \AA$ as Index $(\mathrm{Na} \mathrm{I})=F_{8135-8155} / F_{8180-8200}$, where $F_{8135-8155}$ and $F_{8180-8200}$ are fluxes between 8135 and $8155 \AA$, and between 8180 and $8200 \AA$, respectively. We also calculate the Index(Na I) for the MS stars and giants using the spectra for MS stars and giants from Danks \& Dennefeld (1994) and Covey et al. (2007), respectively. To clarify the criteria for the selection of PMS stars, we use a well-studied sample of the PMS stars in L1641 (Fang et al. 2013a). In Figure 5(a), we show the Index(Na I) for PMS stars and MS stars identified in L1641. In the figure, we also show

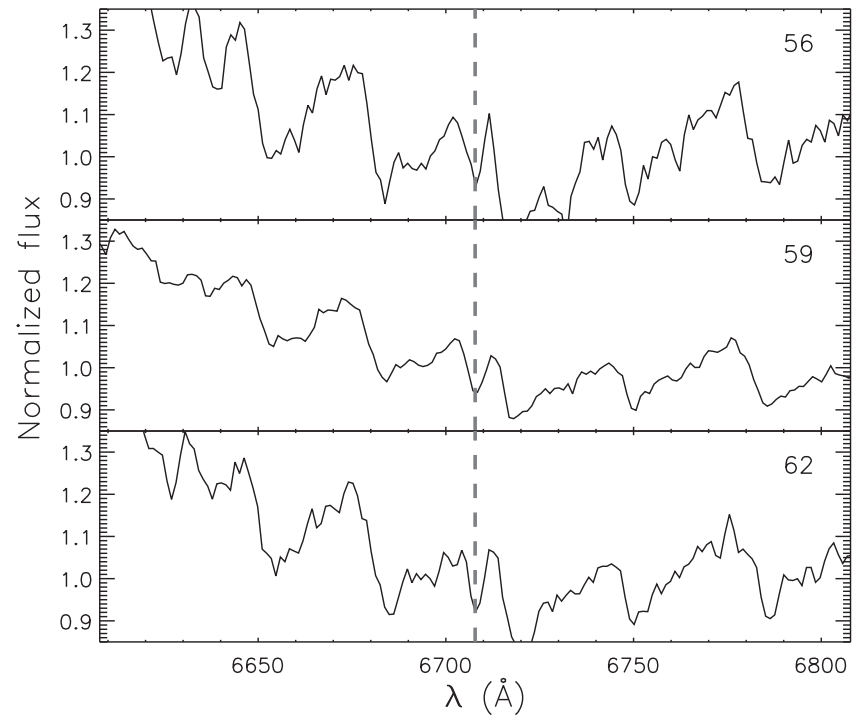

Figure 4. Examples of Li I $\lambda 6708 \AA$ detection with the MMT/Hectospec. The flux is normalized in each panel.

the expected Index(Na I) values for MS stars and giants, which are derived from the spectra for MS stars and giants in the IRTF spectral library (Rayner et al. 2009), and the SDSS spectral templates for MS stars Covey et al. (2007). We note that using Index(Na I) to separate the MS and PMS stars only works for the M-type stars, which is consistent with the findings of Lawson et al. (2009). According to the distribution of the MS stars, PMS stars, and giants in Figure 5(a), we draw the boundaries for selecting PMS stars described as follows. For spectral types between M1 and M3.5, $1.03<\operatorname{Index}(\mathrm{NaI})<1.22$, and for spectral types between M3.5 and M7, $1.03<$ Index $(\mathrm{NaI})<-3.61+0.0657 \times$ Sptnum, where Sptnum is a number corresponding to a spectral type with 70 for M0, 75 for M5, and etc. As a comparison, in Figure 5(b), we show the PMS stars, identified with the $\mathrm{Li}$ I $\lambda 6708 \AA$ absorption, in this work. As expected, most sources are at the PMS boundary but six of them are not. We have checked their spectra. For three of those stars, which show Index(Na I) values simiar to giants, the fringe patterns on their spectra can explain lower Index(Na I) values. The other three stars with large Index(NI) values show clear Li absorption as well as strong Na I doublets at 8183 and $8195 \AA$. A detailed discussion of these sources is presented in Section 4.1. In Figure 5(c), we show the stars without estimated Li I $\lambda 6708 \AA \mathrm{EW}$ due to their noisy spectra. For these stars, we characterize their youth using Index(Na I), in combination with X-ray emission and infrared excess emission.

\subsubsection{X-Ray Emission and Infrared Excess Emission}

In the field shown in Figure 1, we extracted 3,688 XMM $\mathrm{X}$-ray sources from the third $X M M$ Serendipitous Source Catalog (Rosen et al. 2015), and 1616 Chandra X-ray sources from Getman et al. (2005). We matched the X-ray sources to our sources using $1^{\prime \prime}$ tolerance, and found 171 counterparts for our targets. We note that there are more than 100 targets with $\mathrm{X}$-ray sources within radii of $1^{\prime \prime}-3^{\prime \prime}$. We visually check the 2MASS images, and consider those as conterparts to the X-ray sources if there is only one source within $1^{\prime \prime}-3^{\prime \prime}$ from the X-ray source. In this way, we found 107 counterparts in our sample for the X-ray sources. In total, we have 267 sources with X-ray emission from the $X M M$ observations, and an additional 11 


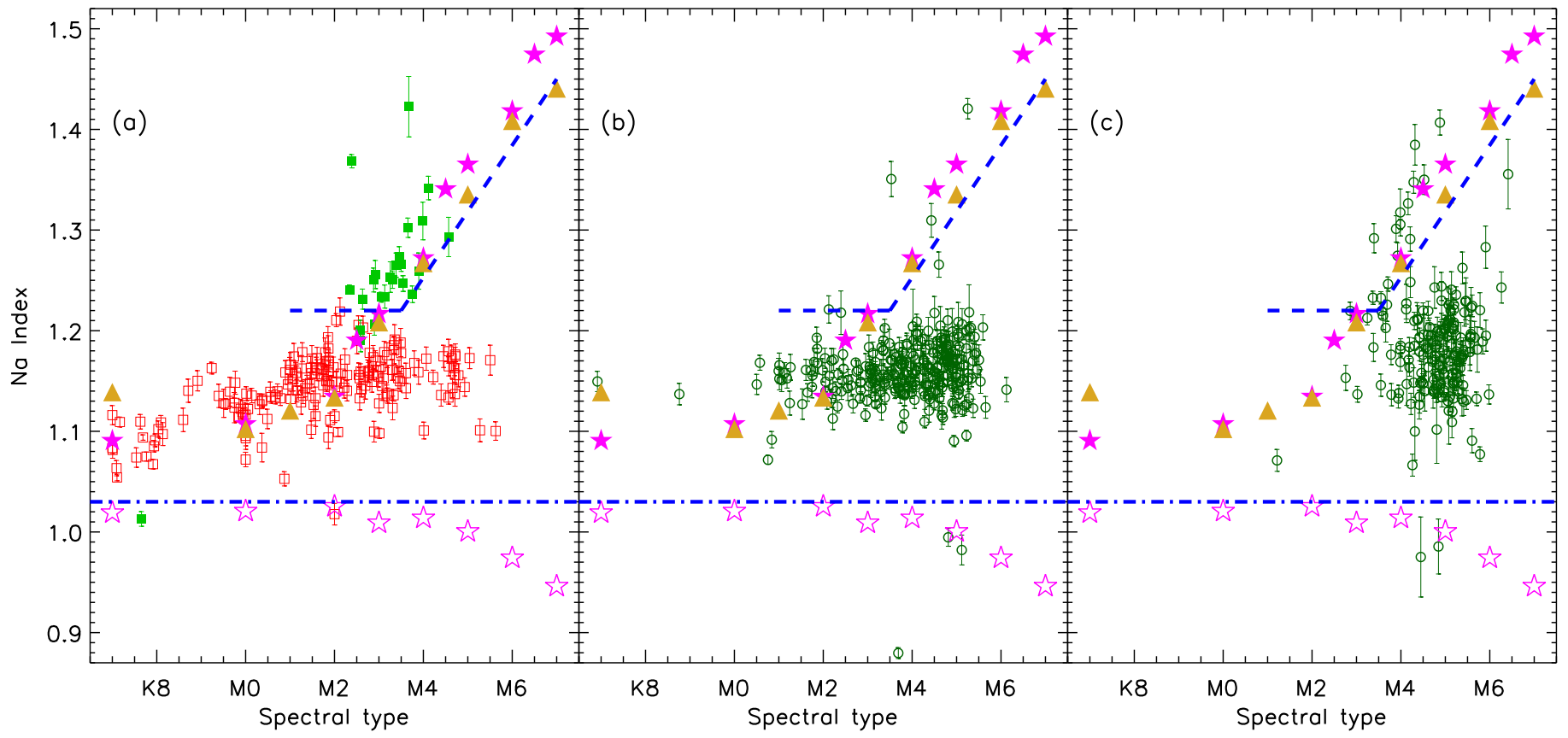

Figure 5. (a) Index(Na I) vs. spectral types for the stars in the field of L1641 (Fang et al. 2013a). The filled squares are for the identified field MS or giant stars in the field of L1641, and the open squares show the PMS stars. The dashed and dashed-dotted lines show the boundaries for MS stars and giants, respectively. The boundaries are defined according to the expected Index(Na I) of MS stars and giants, which are derived using the spectra for MS (filled star symbols) and giants (open star symbols) in the IRTF spectral library (Rayner et al. 2009), and the SDSS spectral templates for MS stars (filled triangles; Covey et al. 2007). (b) Similar to panel (a), but for the PMS stars, which are identified using Li I $\lambda 6708 \AA$ absorption, in this work. (c) Similar to panel (b), but for the stars in this work without knowledge of Li I $\lambda 6708 \AA$ absorption due to their noisy spectra.

sources with X-ray emission from Chandra observations. There are two main contaminators to the YSO catalog selected based on the X-ray data: extragalactic sources and nearby foreground stars. Since we have spectra for each source, extragalactic contaminators can be excluded.

The foreground nearby MS stars can show detectable X-ray emission. We used the tool, Flux Limits from Images from XMM-Newton (FLIX), ${ }^{9}$ to estimate the $5 \sigma$ upper limit of the $0.2-2 \mathrm{keV} X$-ray luminosity in the studied field. We note that the $5 \sigma$ upper limits vary from region to region with a typical value of several $10^{29} \mathrm{erg} \mathrm{s}^{-1}$ at the vicinity of Orion. The typical X-ray luminosity of field MS stars is several $\times 10^{27} \mathrm{erg} \mathrm{s}^{-1}$ for solartype to M-type main-sequence stars (Güdel 2004). According to the $5 \sigma$ upper limit of the $0.2-2 \mathrm{keV}$ X-ray luminosity in the $X M M$ survey of Orion estimated with FLIX, the XMM observations can detect the field MS stars within the distance $\lesssim 50$ pc. Based on the Besançon model of stellar population synthesis of the Galaxy (Robin et al. 2003), we expect only $4 \pm 2$ field MS stars within a distance of $50 \mathrm{pc}$ in the direction of Orion, suggesting that the fraction of contaminators from foreground stars in our YSO catalog, selected from X-ray emission, is negligible.

We also use infrared excess to identify the young stars. A detailed description of characterizing the disk properties of our sample is presented in Section 3.5.

\subsection{Intrinsic Colors of Young Stars}

The extinction of young stars can be determined by comparing the observed colors with the intrinsic colors expected

\footnotetext{
9 FLIX is an online tool provided by the XMM-Newton Survey Science Center (see http://www.ledas.ac.uk/flix/flix3). It provides robust estimates of the X-ray upper limit to a given point in the sky where there are no sources detected in the 3XMMi catalog.
}

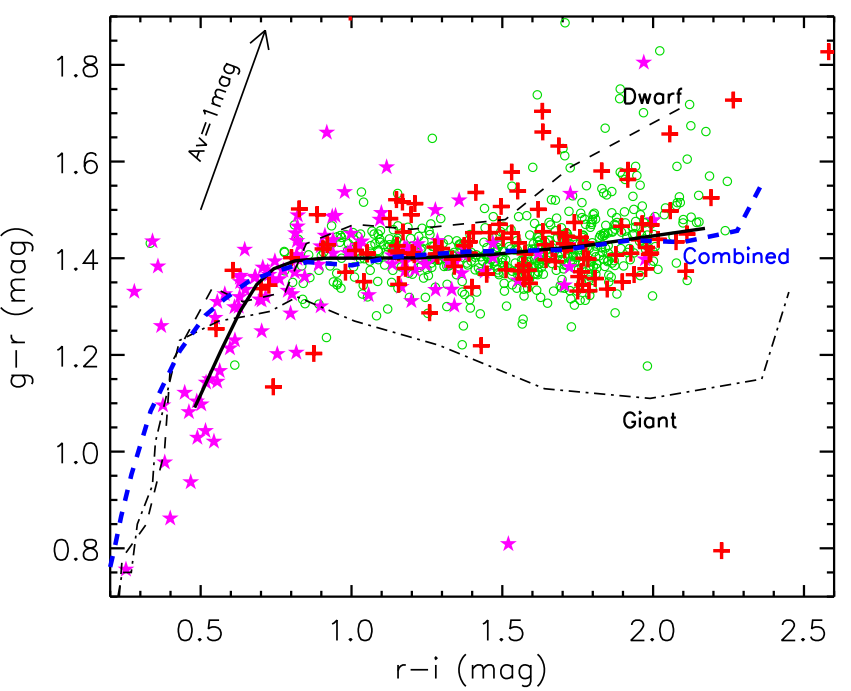

Figure 6. $g-r$ vs. $r-i$ color-color diagram. The open circles show the young stars in this work. The filled star symbols and pluses show the dereddened colors of young stars with low extinction $\left(A_{\mathrm{V}}<0.3\right)$ in Orion OB $1 / \sigma$ Ori, and L1641, respectively. The thick dark solid line shows the empirical colors for young stars used in this work (see Section 3.3). The thin dark dashed line and the dashed-dotted line show the empirical colors for dwarfs and giants, respectively (Covey et al. 2007). The thick blue dashed line shows the synthetic colors using a set of the combined BT-Settl models with solar abundances from Asplund et al. (2009; see Section 3.3).

from their spectral types. In Figure 6, we show the $g-r$ versus $r-i$ color-color diagram for our sources. In the figure, we also show the young stars with low extinction $\left(A_{\mathrm{V}}<0.3\right)$ in Orion OB1, $\sigma$ Ori, and L1641 (Briceño et al. 2005, 2007; Fang et al. 2013a; Downes et al. 2014, 2015; Hernández et al. 2014). Their colors are dereddend using the extinction law from 

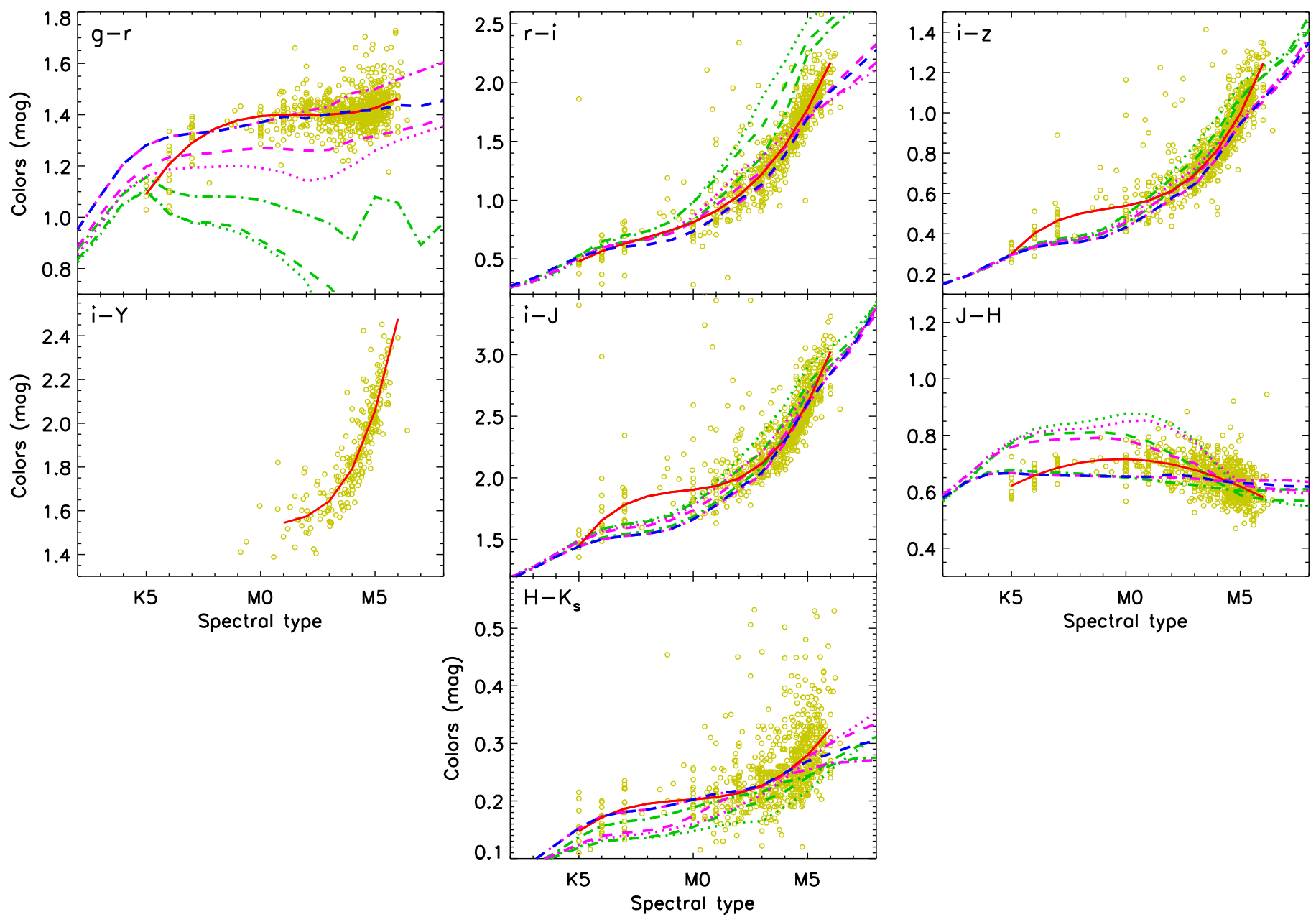

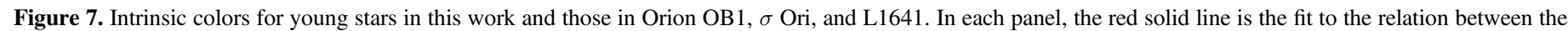

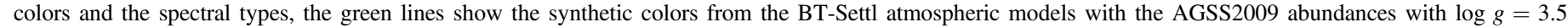

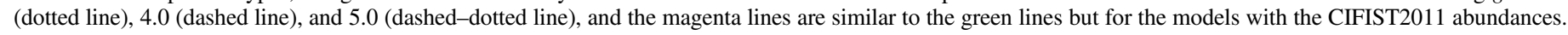
The blue dashed lines show the synthetic colors from the combined BT-Settl models.

Schlafly \& Finkbeiner (2011), a total to selective extinction value typical of interstellar medium dust $\left(R_{\mathrm{V}}=3.1\right)$, and the extinction values in the literature. These young stars in other regions show colors similar to our sources, indicating that the majority of our sources in this work have low or no extinction. As a comparison, in Figure 6, we also show the empirical colors for dwarfs and giants (Covey et al. 2007). We note that there is a shift between the observed colors and the empirical colors for the dwarfs (thin dark dashed line) and giants (thin dark dasheddotted line). In order to derive the extinction properly, we need to construct intrinsic colors for our sources. We use the sources with negligible extinction shown in Figure 6, and extract their griz-band photometric data from SDSS, $J H K_{\mathrm{s}}$-band photometric data from 2MASS. The $Y$-band photometry is only available for the sources in Orion A, and come from Bouy et al. (2014). In Figure 7, we show their colors versus their spectral types. We fit the relations between them using third-order polynomial functions. In Table 2, we list the empirical colors for young stars with spectral types ranging from K5 to M6 from the fitting.

We calculate the synthetic colors using two sets of BT-Settl atmospheric models (Allard et al. 2011, 2012): one with the solar abundances (AGSS2009) from Asplund et al. (2009), and the other with the solar abundances (CIFIST2011) from Caffau et al. (2011). Figure 7 shows the synthetic $g-r$, $r-i, i-z, j-J, J-H$, and $H-K_{\mathrm{s}}$ colors from these models with surface gravity $\log g=3.5,4.0$, and 5.0. While both sets of models are consistent with each other in $i-z$, $j-J, J-H$, and $H-K_{\mathrm{s}}$ colors, the models with the AGSS2009 abundances show the $g-r$ and $r-i$ colors, which are more consistent with the observations than the ones with the CIFIST2011 abundances. To reproduce the observed colors, we construct a set of BT-Settl atmospheric models with the AGSS2009 abundances and using different $\log g$ values for different spectral types: $\log g=5.0$ for the spectral types earlier than M1, $\log g=4.8$ for spectral types between M1 and M3, $\log g=4.6$ for spectral types between M3 and M6, and $\log g=4.5$ for spectral types later than M6. The synthetic colors from this set of models are shown in Figures 6 and 7 , and can better fit the observations than using the atmospheric models with one $\log g$ value. Table 3 lists the synthetic colors and bolometric correction in $J$ band from this set of models. In the table, the conversions from the spectral types to effective temperatures are from Pecaut \& Mamajek (2013) for stars earlier than M4 and from Herczeg \& Hillenbrand (2014) for stars later than M4. In Table 3, we also list the $J$-band bolometric correction Pecaut \& Mamajek (2013, P13), and the ones from Herczeg \& Hillenbrand (2015, H15). Our bolometric corrections are consistent with those in the literature. 
Table 2

Emprical Intrinsic Colors for Young Stars

\begin{tabular}{|c|c|c|c|c|c|c|c|c|}
\hline Spt & $\begin{array}{l}\text { Teff } \\
(\mathrm{K})\end{array}$ & $\begin{array}{l}g-r \\
(\mathrm{mag})\end{array}$ & $\begin{array}{l}r-i \\
(\mathrm{mag})\end{array}$ & $\begin{array}{l}i-z \\
(\mathrm{mag})\end{array}$ & $\begin{array}{l}i-Y \\
(\mathrm{mag})\end{array}$ & $\begin{array}{l}i-J \\
(\mathrm{mag})\end{array}$ & $\begin{array}{c}J-H \\
(\mathrm{mag})\end{array}$ & $\begin{array}{c}H-K s \\
(\mathrm{mag})\end{array}$ \\
\hline K5 & 4140 & 1.09 & 0.48 & 0.30 & $\ldots$ & 1.45 & 0.62 & 0.15 \\
\hline K6 & 4020 & 1.21 & 0.57 & 0.40 & $\ldots$ & 1.65 & 0.66 & 0.17 \\
\hline K7 & 3970 & 1.29 & 0.63 & 0.46 & $\ldots$ & 1.78 & 0.68 & 0.19 \\
\hline K8 & 3940 & 1.35 & 0.69 & 0.50 & $\ldots$ & 1.85 & 0.70 & 0.20 \\
\hline K9 & 3880 & 1.38 & 0.74 & 0.52 & $\ldots$ & 1.88 & 0.71 & 0.20 \\
\hline M0 & 3770 & 1.39 & 0.81 & 0.54 & $\ldots$ & 1.90 & 0.72 & 0.20 \\
\hline M1 & 3630 & 1.40 & 0.91 & 0.57 & 1.54 & 1.93 & 0.71 & 0.21 \\
\hline M2 & 3490 & 1.40 & 1.04 & 0.61 & 1.57 & 2.00 & 0.70 & 0.21 \\
\hline M3 & 3360 & 1.40 & 1.22 & 0.69 & 1.64 & 2.11 & 0.68 & 0.23 \\
\hline M4 & 3160 & 1.41 & 1.46 & 0.82 & 1.79 & 2.31 & 0.65 & 0.25 \\
\hline M5 & 2980 & 1.43 & 1.78 & 1.00 & 2.06 & 2.60 & 0.62 & 0.28 \\
\hline M6 & 2860 & 1.46 & 2.17 & 1.25 & 2.48 & 3.02 & 0.58 & 0.32 \\
\hline
\end{tabular}

\subsection{Determining Stellar Properties}

We derive the extinction for our sources using the empirical colors listed in Table 2 for the ones with spectral types earlier than M6, and the synthetic colors in Table 3 for those with spectral types later than M6. Using the intrinsic colors of $g-r$, $r-i, i-z, i-j$, we derive mean visual extinctions $\left(A_{V}\right)$ for individual sources employing the extinction law from Schlafly \& Finkbeiner (2011) and a total to selective extinction ratio typical of interstellar medium dust $\left(R_{\mathrm{V}}=3.1\right)$. We converted the spectral types to effective temperatures using the relation in Table 3, and derive the stellar luminosities for our sources using the $J$-band bolometric correction for the corresponding spectral types calculated in this work. The stellar luminosities $\left(L_{\star}\right)$ are then calculated as follows.

$$
\begin{aligned}
M_{\mathrm{bol}} & =B C_{J}+m_{J}-5 \times \log \left(\frac{d}{10 \mathrm{pc}}\right) \\
\frac{L_{\star}}{L_{\odot}} & =10^{\frac{M_{\mathrm{bol}, \odot}-M_{\mathrm{bol}}}{2.5}}
\end{aligned}
$$

where $B C_{J}$ is the the bolometric correction in $J$ band, $m_{J}$ is the dereddened apparent magnitude in $J$ band, $d$ is the distance in units of parsecs, $M_{\mathrm{bol}, \odot}$ is the bolometric magnitude of the Sun. Here we take $M_{\mathrm{bol}, \odot}=4.755$ (Mamajek 2012).

\subsection{Characterizing Disk Properties}

We characterize the disk properties by comparing the model atmosperes from the BT-Settl models with the observed SEDs. The typical way is to compare the infrared spectral slopes with the expected slopes at the same wavelength ranges from purely photospheric emissions (Luhman et al. 2008). The infrared spectral slopes, defined as $\alpha=d \log \left(\lambda F_{\lambda}\right) / d \log (\lambda)$, are calculated with the dereddened photometry in each band. We compute two sets of infrared spectral slopes, $\alpha_{3.6-8}$ and $\alpha_{3.6-24}$, corresponding to the spectral range of [3.6] to [8.0] and [3.6] to [24], respectively. Table 6 lists the two infrared spectral slopes of the sources with detection in the corresponding infrared bands. In Figure 8, we show the infrared spectral slopes, $\alpha_{3.6-8}$ and $\alpha_{3.6-24}$ versus the spectral types, for our sources. As a comparison, we calculate the spectral slopes (see Figure 8) of the BT-Settl atmospheric models (Allard et al. 2012), assuming a $10 \%$ uncertainty in Spitzer photometry. Stars with the infrared spectral slopes steeper than the slopes of photospheric emissions are considered to be diskless. For sources with shallower infrared spectral slopes, we visually examine their SEDs. The sources that show infrared excess at more than a $3 \sigma$ confidence level are considered to have disks. A total of 185 sources belong to the disk population. In addition, there are 119 sources without detection in all four IRAC bands. We visually compare their SEDs, constructed from the available Spitzer and WISE photometric data, to their photospheric emission. Among the 119 sources, 9 sources show infrared excess at more than a $3 \sigma$ confidence level, and are considered to have disks. In Table 6, we list the disk property of each source.

The young stars can be grouped into weak-line $\mathrm{T}$ Tauri stars (WTTS) or classical T Tauri stars (CTTS) based on their $\mathrm{H} \alpha$ EW. WTTSs have stopped accretion, and show weak and narrow $\mathrm{H} \alpha$ emission lines in the spectra, while CTTSs are still accreting, and present strong and broad $\mathrm{H} \alpha$ emission lines. We divide the YSOs into WTTS or CTTS using the criteria described in Fang et al. (2009), in which a star is classified as a CTTS if $\mathrm{EW}(\mathrm{H} \alpha) \geqslant 3 \AA$ for $\mathrm{K} 0-\mathrm{K} 3$ stars, $\mathrm{EW}(\mathrm{H} \alpha) \geqslant 5 \AA$ for $\mathrm{K} 4$ stars, $\mathrm{EW}(\mathrm{H} \alpha) \geqslant 7 \AA$ for $\mathrm{K} 5-\mathrm{K} 7$ stars, $\mathrm{EW}(\mathrm{H} \alpha) \geqslant 9 \AA$ for M0-M1 stars, $\mathrm{EW}(\mathrm{H} \alpha) \geqslant 11 \AA$ for M2 stars, $\mathrm{EW}(\mathrm{H} \alpha) \geqslant 15 \AA$ for M3-M4 stars, EW $(\mathrm{H} \alpha) \geqslant 18 \AA$ for M5-M6 stars, and EW $(\mathrm{H} \alpha) \geqslant 20 \AA$ for M7-M8 stars. Table 6 list the $\mathrm{H} \alpha E W s$ of each source and its accretion property. However, we must stress that using $\mathrm{EW}(\mathrm{H} \alpha)$ to distinguish WTTSs and CTTSs can fail to distinguish some stars that have low accretion rates, strong chromospheric activity, sky contamination, or selfabsorptions of the $\mathrm{H} \alpha$ line.

\section{Results \\ 4.1. A Census of PMS Stars}

We identify PMS stars mainly based on the LiI $\lambda 6708 \AA$ absorption. With this criterion, a sample of 447 stars are classified as PMS stars. For others without estimates of the EWs of the Li I $\lambda 6708 \AA$ absorption line, we use the Index(Na I) to select the PMS stars as described in Section 3.2.1, which works for stars with spectral types later than M1. With this criterion, an additional 164 stars are classified as PMS stars. We also include 64 additional stars that show X-ray emission, and 27 additional stars with infrared excess emission. A total of 691 sources are classified as PMS stars according to the above criteria. Table 6 lists these stars, as well as the criteria to classify them as the PMS stars.

We use the Index(Na I) to assess the contamination in our PMS sample from young field stars, which may show weak 
Table 3

Synthetic Colors for Young Stars

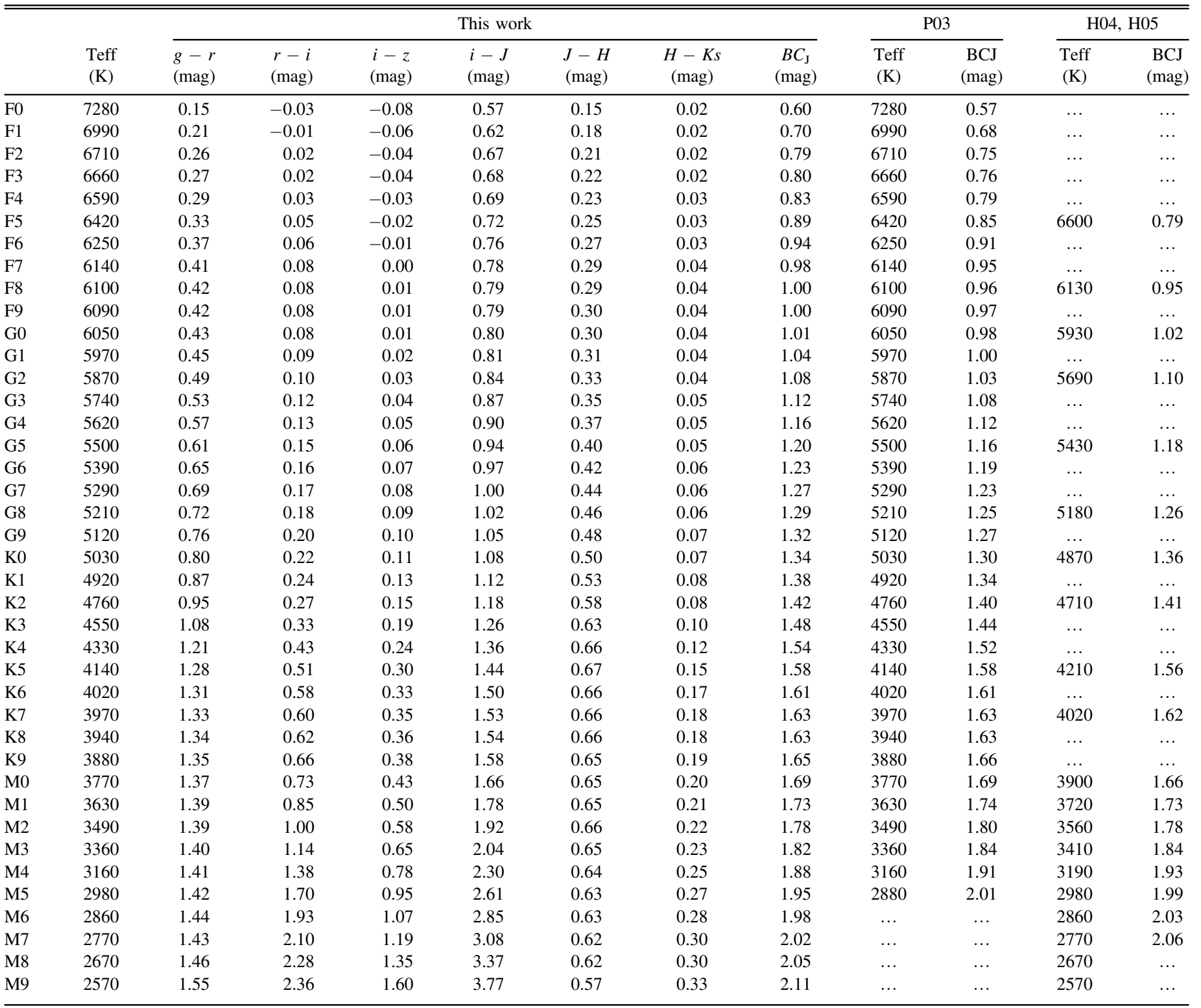

Li I $\lambda 6708 \AA$ absorption lines. In our sample, there are 447 sources with estimated EWs of the Li I $\lambda 6708 \AA$ absorption line, and $63 \%$ of them $(283 / 447)$ have the Index(Na I) in Table 6. As noted in Section 3.2.1, there are three sources, ID 156, 438, and 572, in Table 6, that are located outside the PMS boundary in Figure 5(b) and show Index(Na I) similar to the field dwarfs. However, for two sources, ID 156 and 438, the strengths of their $\operatorname{LiI} \lambda 6708 \AA$ absorption lines are consistent with other young stars in Orion (see, e.g., Fang et al. 2013a). Hence, it is unclear why they show the strong Na I doublets. For the source 572, its Li I $\lambda 6708 \AA$ absorption line is weak ( $\mathrm{EW} \sim 0.2$ ), and could be a young field dwarf. Therefore, we expect that the contamination in our sample from the field dwarfs is not significant.

\subsection{Stellar Properties}

We derive the extinction of individual sources in the way described in Section 3.4. The typical uncertainty for our $A_{V}$ measurements is around $0.3 \mathrm{mag}$, estimated from the sources without extinction in our sample. The resulting visual extinctions are listed in Table 6. With the derived effective temperatures and bolometric luminosities using the method described in Section 3.4, assuming a distance of $414 \mathrm{pc}$, we place the stars in the H-R diagram in Figure 9. Most of our sources lie between the 0.1 and $3 \mathrm{Myr}$ isochrones. We use a distinct symbol (open square symbols) for an "exotic" object that is apparently subluminous and an extremely low-mass object (see Section 4.3.1 and Section 4.3.2). We derive the masses and ages of the stars using the PMS evolutionary tracks from Baraffe et al. (2015). For the stars above the youngest isochrone $(\sim 0.5 \mathrm{Myr})$ from the evolutionary models, their masses are estimated using the $0.5 \mathrm{Myr}$ isochrone since the low-mass stars are evolving vertically in the H-R diagram during the first several Myr. In Table 6, we list the stellar masses and ages of the stars. Seventeen objects in our sample have masses less than $0.075 M_{\odot}$, and are brown dwarfs. Among them, the source 414 has the minimum mass $\left(\sim 0.018 M_{\odot}\right)$. In 


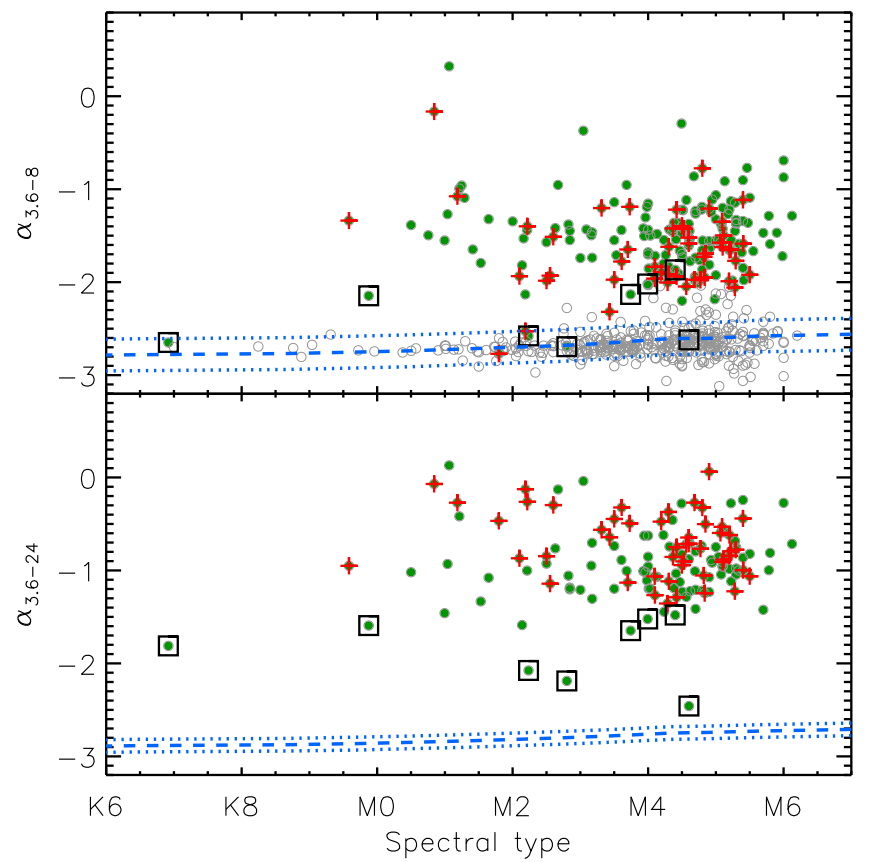

Figure 8. Dereddened Spitzer infrared spectral slopes, $\alpha_{3.6-8}$ and $\alpha_{3.6-24}$ vs spectral type. In each panel, the open gray circles show the diskless stars, and the filled circles are for the sources with disks. The plus symbols mark the transition disks, and the open squares are for the other evolved disks in our sample (see Section 4.3.3 and Figure 16). The dashed line shows the infrared spectral slope of the photospheric emission calculated with the BT-Settl atmospheric models, and the dotted lines are the $1 \sigma$ standard deviation, assuming a $10 \%$ uncertainty in Spitzer photometry.

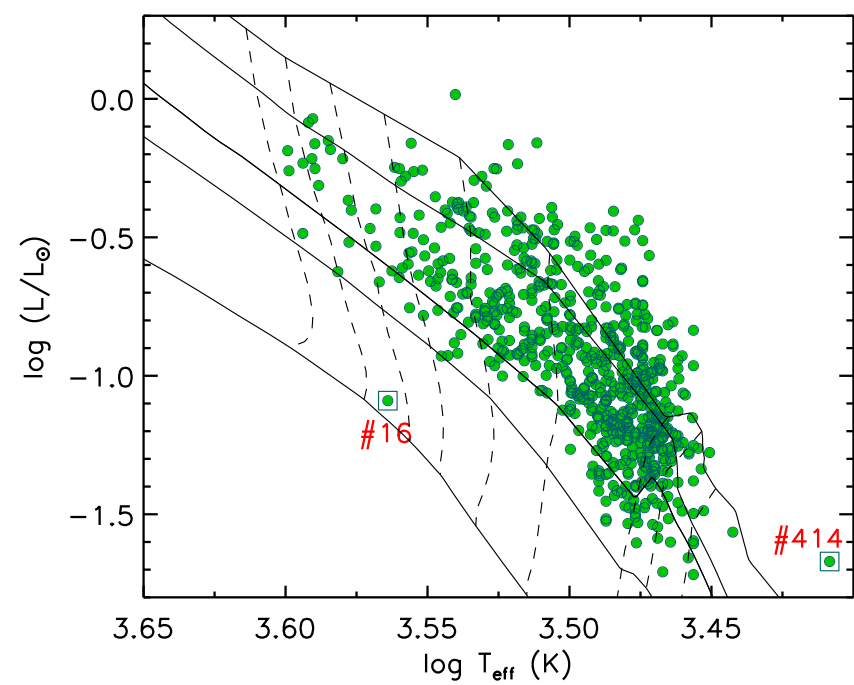

Figure 9. H-R diagram for the PMS stars (filled circles) in this work. The open squares mark the two interesting objects in our sample (see Section 4.3.1 and 4.3.2). The solid lines show the isochrones at ages of $0.5,1,3,5,, 10$, and $30 \mathrm{Myr}$, respectively, from Baraffe et al. (2015). The dashed lines present the evolutionary tracks of young stellar/substellar objects with masses of 0.06 , $0.08,0.1,0.2,0.3,0.4,0.5,0.6$, and $0.7 M_{\odot}$, respectively.

Figure 10, we show the mass and age distributions of our sample. The median mass and age of our sample is $\sim 0.15 M_{\odot}$ and $\sim 1.4 \mathrm{Myr}$, respectively. In Figure 11, we show the age distributions of the young stars with and without disks. Both populations show a similar age distribution with most of the stars being younger than $2.5 \mathrm{Myr}$. The median ages of the two populations are 1.3 and $1.6 \mathrm{Myr}$ for diskless and disk populations, respectively.
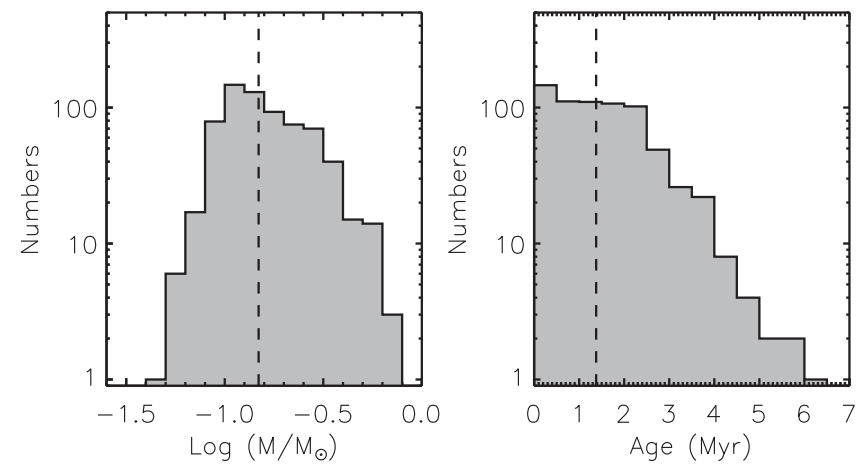

Figure 10. Mass (left) and age (right) distributions of the young stars. The dashed lines shows the median mass $\left(\sim 0.15 M_{\odot}\right)$ and age $(\sim 1.4 \mathrm{Myr})$ of our sample, respectively.

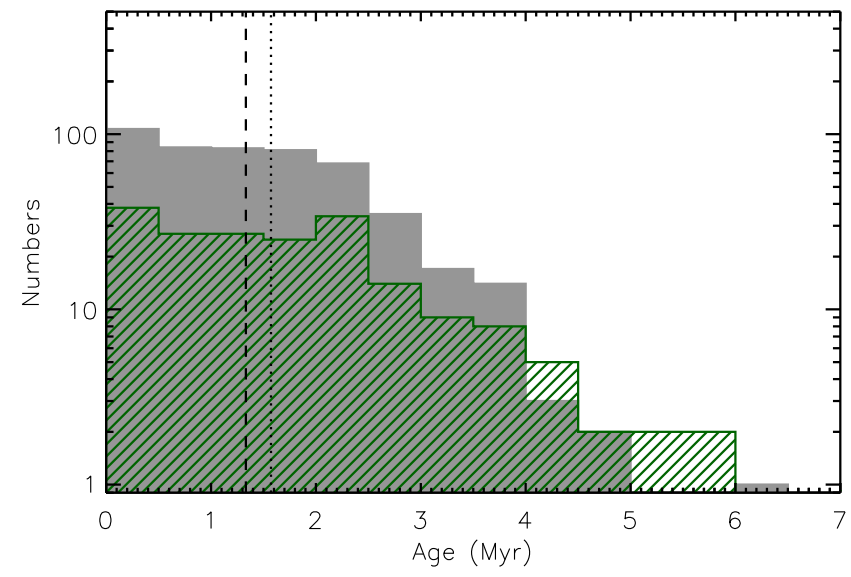

Figure 11. Age distributions of the young stars with disks (line-filled histogram) and without disks (gray-color filled histogram). The median ages of the two populations, 1.6 and $1.3 \mathrm{Myr}$, respectively, are shown with the dotted line and the dashed line.

We must stress that the stellar ages derived from the H-R diagram are dependent on the distance that we use. Here, we assume that the foreground population is associated with the Orion molecular cloud, and use the same distance $(414 \mathrm{pc})$ as Orion, which is supported by the fact that the foreground population and the Orion molecular cloud show similar kinematics (see Section 5.1).

\subsection{Disk Properties}

\subsubsection{A Subluminous Object}

In the H-R diagram (see Figure 9), one source (ID 16 in Table 6) appears to be subluminous compared to others with similar spectral types. In its spectrum, we detect an Li I $\lambda 6708 \AA$ absorption line, indicating that it is a PMS star. The isochrone age of this object is $\sim 23 \mathrm{Myr}$. However, its optical spectrum shows numerous strong emission lines. Among them (see the left panel in Figure 12), the $\mathrm{H} \alpha$ is the strongest one with an EW of $-134 \AA$. Such strong emission lines in the spectrum of Source 16 indicate that it should be much younger than its isochronal age ( 23 Myr). Its SED shows the strong infrared excess emission (see the right panel in Figure 12), suggesting that it is surrounded by a disk. Similar young stars have been discovered in our previous spectroscopic surveys in Orion (Fang et al. 2009, 2013a), and also found in other regions, e.g., the Lupus 3 dark cloud, Taurus, and $\epsilon$ Cha (Comerón et al. 2003; White \& Hillenbrand 2004; Fang et al. 2013b). One promising 

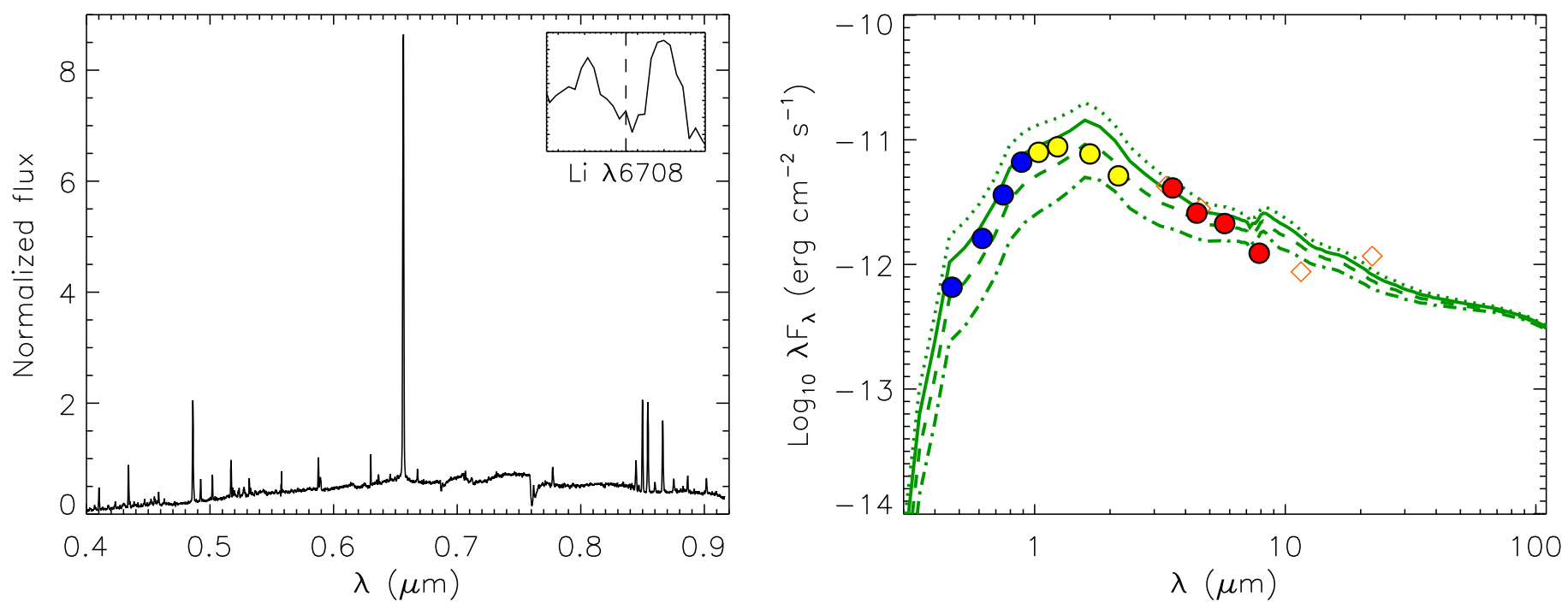

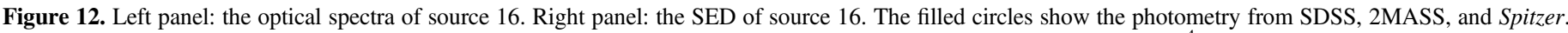

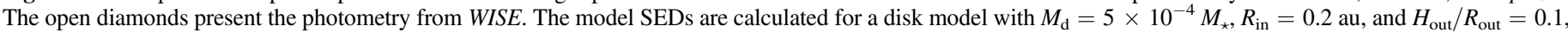

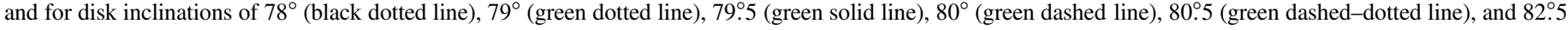
(black dashed-dotted line), respectively.

hypothesis for these exotic objects is that they are harboring disks with high inclinations, and the light seen mainly comes from photons scattered off the disk surface and is therefore much reduced. The optical emission lines may arise in outflows or disk winds with emitting areas much larger than the central star, which allows at least part of the line fluxes to reach us relatively unattenuated. We employ the radiative transfer code RADMC-3D (Dullemond et al. 2012) to model the SED of Source 16. We set the stellar effective temperature to $3664 \mathrm{~K}$ (M0.8) and stellar radius $1.3 R_{\odot}$, corresponding to a PMS star with a mass of $\sim 0.4 M_{\odot}$ and an age of 2 Myr (Baraffe et al. 2015). We set the outer disk radius $\left(R_{\text {out }}\right)$ to $100 \mathrm{au}$, and assume a pressure scale height $\left(H_{\mathrm{P}}\right)$ that varies as a power law with the radius $(R), H_{\mathrm{P}} /$ $R \propto R^{1 / 7}$. We vary the disk mass $\left(M_{\mathrm{d}}\right)$ from $10^{-4} M_{\star}$, $5 \times 10^{-4} M_{\star}, 1 \times 10^{-3} M_{\star}, 5 \times 10^{-3} M_{\star}$, to $10^{-2} M_{\star}$, the inner disk radius $\left(R_{\text {in }}\right)$ from $0.1,0.2,0.3$, to $0.4 \mathrm{au}, H_{\text {out }} / R_{\text {out }}\left(H_{\text {out }}\right.$ : the pressure scale height at $R_{\text {out }}$ ) from $0.1,0.2$, to 0.3 , and disk inclinations from $60^{\circ}$ to $83^{\circ}$. We find that the SED of Source 16 can be reproduced by a model with parameters of $H_{\text {out }} /$ $R_{\text {out }}=0.1, M_{\mathrm{d}}=10^{-4} M_{\star}-1 \times 10^{-3} M_{\star}$, and $R_{\text {in }}=0.1-0.4 \mathrm{au}$, and an inclination of $\sim 79^{\circ}-82^{\circ}$ (see Figure 12 for a representative model). A lower or higher disk inclination can lead to significantly over-predicted or under-predicted fluxes at short wavelengths, respectively (see Figure 12).

The prominent emission lines in the spectrum of ID 16 include Balmer lines, He I lines at $5876 \AA$ and $6678 \AA$, [O I] lines at $5577 \AA$ and $6300 \AA$, Ca II infrared triplet (8498, 8542, $8662 \AA$ ), as well as many other lines. Such a spectrum rich in emission lines is similar to that of EX Lup, an M0-type young star and the prototype of the EXor variable star (Sicilia-Aguilar et al. 2015a). A detailed identification of all the lines and a comparison of ID 16 to EX Lup would require spectra with high spectral resolutions. If the underluminosity of ID 16 is due to the occultation and scattering of photospheric emission by a highly inclined disk, we would expect that the EWs of the accretion-related lines are simialr to the ones typical to $\mathrm{T}$ Tauri stars since they are formed in the magnetospheric infall flows, which are close to the stellar surface and should be similarly occulted. We notice that the source shows the normal He I $\lambda 6678 \AA$ line, but $\sim 4$ times higher
EWs of the He I $\lambda 5876 \AA$ line, and $\sim 10$ times higher EWs of the Ca II infrared triplet, compared with other CTTSs in Orion (see, e.g., Fang et al. 2009). Both He I emission lines and Ca II infrared triplet are related to accretion activities. The large EWs of these lines could be explained if ID 16 is actively accreting. The spectrum of ID 16 also shows [O I] lines at $5577 \AA$ and $6300 \AA$ with $E W s$, which are also $\sim 10$ times higher than the ones typical to T Tauri stars (Simon et al. 2016). However, these [O I] lines could be contaminated by telluric [O I] emission lines since our spectral resolution is low. In the spectrum of ID 16, we did not clearly detect the the [S II] emission lines at $6716 \AA$ and $6731 \AA$, but may see two peaks near them. The [S II] lines are thought to arise in jets emerging from accreting YSOs. New spectral data with high spectral resolution and a signal-to-noise ratio are needed to identify them explicitly. Here, we must stress that the source ID 16 could also be a young star with a distance much larger than Orion. In this case, we do not need a highly inclined disk to reproduce its SED, and the distance of ID 16 could be $\sim 1 \mathrm{kpc}$, if its age is around $1 \mathrm{Myr}$.

\subsubsection{The Disk around an Extremely Low-mass Object}

In our sample, Source 414 has the latest spectral type. Its spectral type in this work is $\sim \mathrm{M} 9 \pm 1$, which is consistent with the one (M8) in Hillenbrand et al. (2013) given the uncertainties. In the H-R diagram, this object is above the youngest PMS isochrone ( $\sim 0.5 \mathrm{Myr}$ ) from Baraffe et al. (2015). Assuming a $0.5 \mathrm{Myr}$ isochrone, the mass of a source with a spectral type M8-M9 is around $0.018-0.030 M_{\odot}$. However, the mass of $0.018-0.030 M_{\odot}$ should be considered to be the upper limit for Source 414, since the effective temperatures of young extremely low-mass objects decrease during their evolution (Baraffe et al. 2015). In Figure 13, we show the SED of this object. Its SED shows infrared excess emission in all four IRAC bands, suggesting that it possesses a disk. We employ the radiative transfer code RADMC-3D to model the SED of the source 414 . We set the stellar effective temperature to $2570 \mathrm{~K}$ (M9) and the stellar radius to $0.84 R_{\odot}$, and a disk mass to $1 \times 10^{-2} M_{\star}$, and the inner edge to the dust sublimation radius, set $R_{\text {out }}$ to $50 \mathrm{au}$, and assume $H_{\mathrm{P}} / R \propto R^{1 / 7}$. We vary $\left(M_{\mathrm{d}}\right)$ from 


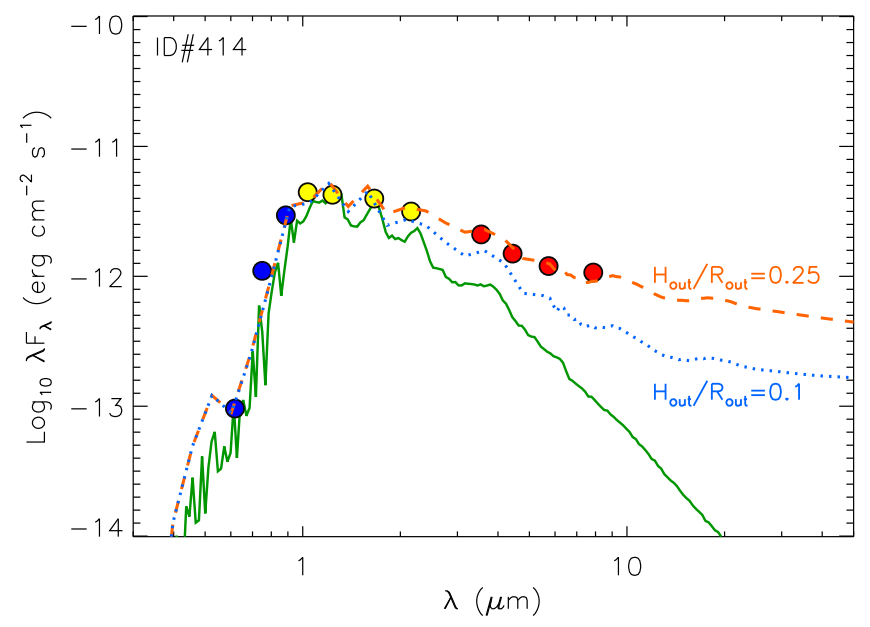

Figure 13. SED of an extremely low-mass object (Source 414). The filled circles show the photometry from SDSS, 2MASS, and Spitzer. The model SEDs are calculated for a disk model with $M_{\mathrm{d}}=1 \times 10^{-2} M_{\star}, H_{\text {out }} /$ $R_{\text {out }}=0.25$ (dashed line) and 0.1 (dotted line), and for a disk inclination of $40^{\circ}$.

$10^{-4} M_{\star}, 5 \times 10^{-4} M_{\star}, 1 \times 10^{-3} M_{\star}, 5 \times 10^{-3} M_{\star}$, to $10^{-2} M_{\star}$, $H_{\text {out }} / R_{\text {out }}$ from $0.1,0.15,0.2,0.25$,to 0.3 , and and disk inclinations from $0^{\circ}$ to $80^{\circ}$. We find that a flaring disk model with $H_{\text {out }} / R_{\text {out }}=0.25-0.3, \quad M_{\mathrm{d}}=5 \times 10^{-4} M_{\star}-1 \times 10^{-2} M_{\star}$ and an disk inclination $\lesssim 50^{\circ}$ can reproduce the SED of Source 414. In Figure 13, we compare the model SED with $H_{\text {out }} / R_{\text {out }}=0.25$ (a flaring disk model) with the one with $H_{\text {out }} /$ $R_{\text {out }}=0.1$ (a flat disk model). Both flat and flared disks have been found around brown dwarfs (Apai et al. 2005; Pascucci et al. 2009).

\subsubsection{Evolved Disks}

In our sample, 689 sources have infrared photometry in at least three IRAC or WISE bands and can be classified as diskless or harboring disks. Among them, 72\% (495/689) are diskless, and the rest are disk sources. Among the disk population, we select transition disk (TD) candidates based on colors as shown in Figure 14: $[8.0]-[24] \geqslant 2.5$ and $K_{\mathrm{s}}-[5.8] \leqslant 0.56+([8.0]-$ $[24]) \times 0.15$. For individual candidates, we compare their SEDs with their model photospheric emissions, as well as the median SEDs of L1641 CTTSs (Fang et al. 2013a), corresponding to their spectral types. A total of 49 sources are confirmed as TD objects, which show very weak or no infrared excess at near-infrared wavelengths and shorter IRAC bands, but strong excess emission at mid-infrared and longer wavelengths. Among them, 12 TDs have already been described in the literarure (Fang et al. 2013a; Kim et al. 2013). In Figure 15, we show the SEDs of the 37 new TDs in this work.

In our sample, we also found eight sources, which were detected in $24 \mu \mathrm{m}$, and showed much weaker infrared excess emission than a typical TD. Their infrared spectral slopes and colors are smaller than other sources (see Figures 8 and 14). In Figure 16, we show the SEDs of those sources. Among these sources, one object (ID 666) shows the depleted infrared excess emission through the four IRAC bands to $24 \mu \mathrm{m}$, and thus could be a globally depleted disk candidate, which can be produced when disks are deficient in small dust grains (Currie et al. 2009; Sicilia-Aguilar et al. 2011). In contrast to Source 666, the SEDs of the objects 279,570 , and 685 start to show the infrared excess emission from $8 \mu \mathrm{m}$, while the others show infrared excess only at $24 \mu \mathrm{m}$. The weak infrared excess emission of the seven sources

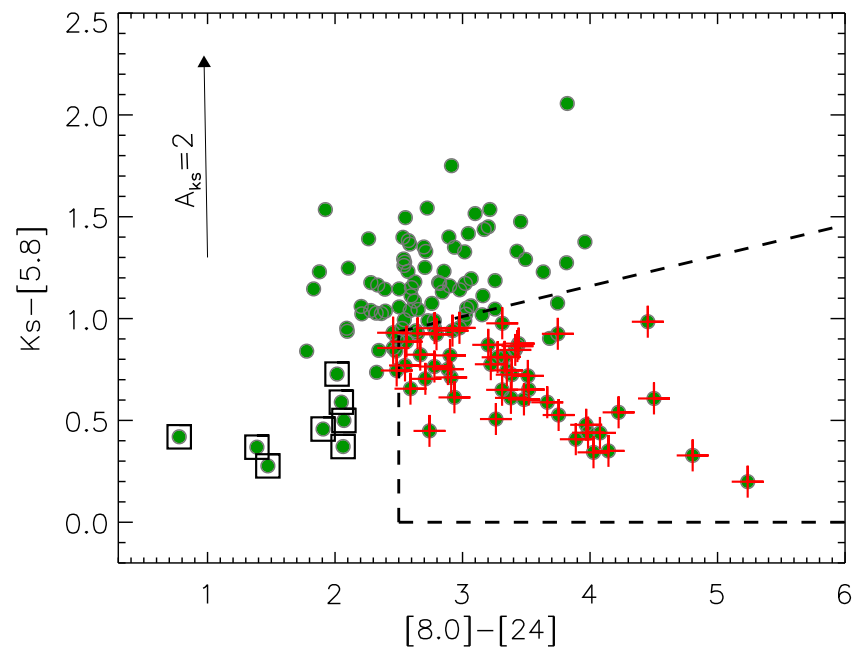

Figure 14. [8.0]-[24] vs. $K_{\mathrm{s}}-[5.8]$ color-color diagram. The dashed lines enclose the region for selecting transition disks. The pluses mark the transition disks, and the open squares show the other evolved disks in our sample (see Section 4.3.3 and Figure 16). The large arrow shows the reddening vector with $K_{\mathrm{s}}$-band extinction of $2 \mathrm{mag}$.

indicates that their inner disks have been more strongly dissipated than a typical TD in the same region. Without the data at farinfrared bands, it is unknown if these sources are TDs with a big inner hole, or young debris disks. We also found two similar sources in L1641 (Fang et al. 2009, 2013a), one of which shows strong excess emission at 70 and $160 \mu \mathrm{m}$ (Source 069001 in Stutz et al. 2013), suggesting that this object is a TD with a big inner hole. Similar objects have been found in the Tr 37 cluster (SiciliaAguilar et al. 2013, 2015b).

In Figure 17, we show the age distributions of the three populations. The median ages of the sources with optically thick disks $\left(\alpha_{3.6-8} \geqslant-1.8\right)$, evolved disks, and without disks are $\sim 1.4 \mathrm{Myr}, 1.8 \mathrm{Myr}$, and 1.4 Myr, respectively. We note that the median age of the evolved disk systems is slightly larger than the other two populations. However, since each population shows a very broad distribution, the difference among their median ages is not significant.

\subsubsection{Accretion in Disks at Different Evolutionary Stages}

In Figure 18(a), we compare the $\mathrm{H} \alpha$ EWs for sources with TDs, optically thick disks $\left(\alpha_{3.6-8} \geqslant-1.8\right)$, or without disks. It can be noted that the YSOs with optically thick disks usually present strong $\mathrm{H} \alpha$ emission, while diskless YSOs show weak $\mathrm{H} \alpha$ emission. However, for TDs, about half show no accretion, while a small fraction of them show strong accretion. In Figure 18(b), we display the distribution of the logarithmic ratio between the observed $\mathrm{H} \alpha \mathrm{EW}$ and the EW threshold for the three populations shown in Figure 18(a). Here, the EW threshold is the one used to classify the YSOs into CTTSs or WTTSs, and is the spectral type dependent (see Section 3.5). According to these EW thresholds, $73 \% \pm 9 \%$ of YSOs with optically thick disks are accreting, while only $46 \% \pm 7 \%$ of TDs are accretors. If we define "strong accretion" sources to have $\mathrm{H} \alpha$ EWs greater than twice the EW thresholds, $25 \% \pm 7 \%$ of TDs belong to this group. For the YSOs with optically thick disks, this fraction is $45 \% \pm 10 \%$. The accretion properties among the different types of disks are consistent with our previous studies (Fang et al. 2009, 2013a; Sicilia-Aguilar et al. 2010, 2013). For the eight evolved disks 


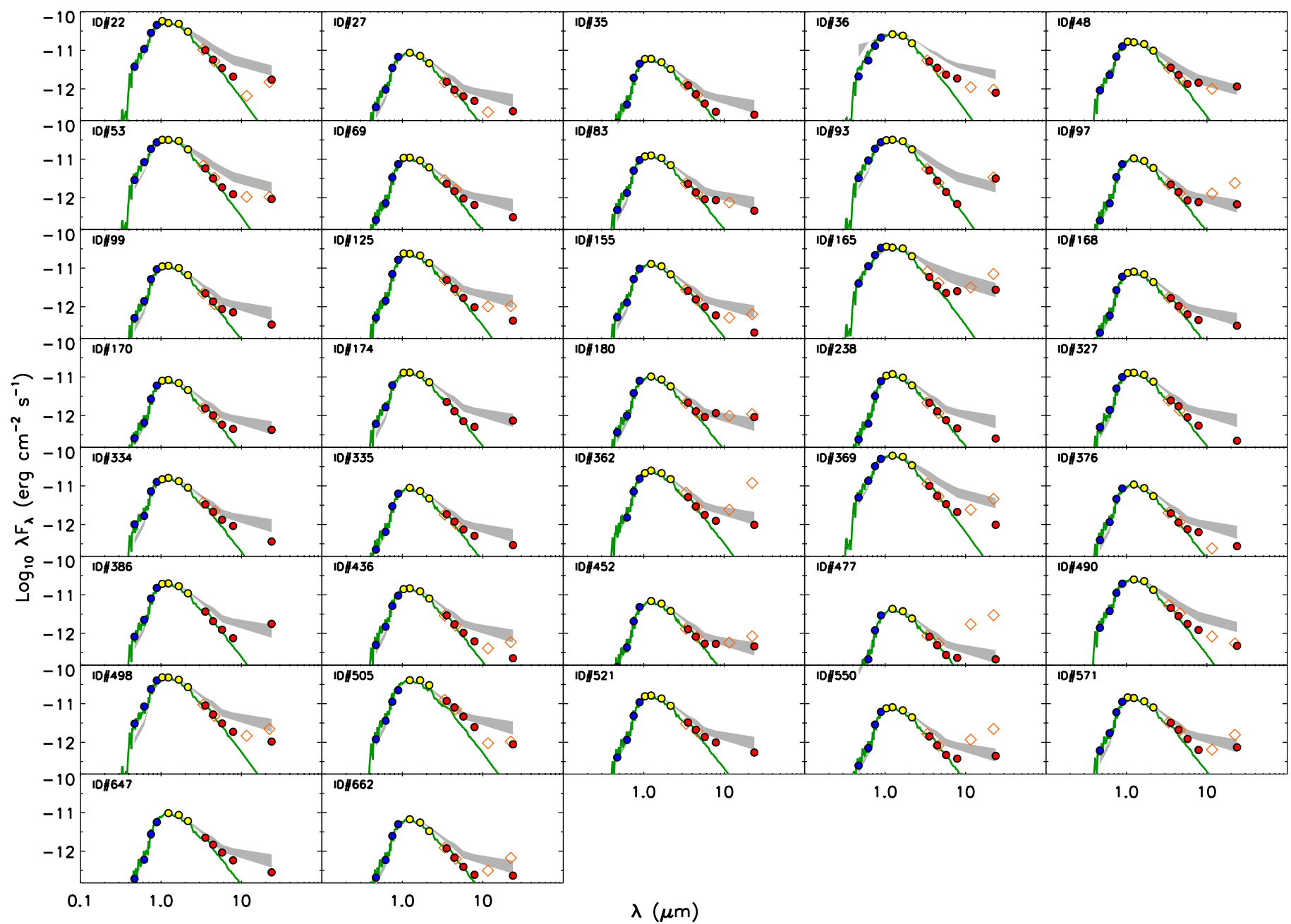

Figure 15. SEDs of newly confirmed YSOs with transition disks. The circles show the photometry in different bands. The open diamonds are the photometry from WISE. In each panel, the green solid curve indicates the photospheric emission level, and the gray-color filled region shows the upper and lower quartiles of the median SEDs of L1641 CTTSs with the spectral types similar to our sources.

shown in Figure 16, seven of them have estimates of $\mathrm{H} \alpha E W s$, six of them show no accretion, and one (499) shows $\mathrm{H} \alpha$ EW, which is just above the threshold to be classified as a CTTS. Therefore, the fraction of accretors among these sources should be less than 14\%, suggesting that these objects are much more evolved than TDs.

\section{Discussion}

\subsection{Is the Foreground Population Associated with the Orion A Cloud?}

Tobin et al. (2009) performed a kinematic survey of 1613 stars, which span from NGC 1977 to L1641N. Their study suggests that the kinematics of young stars are consistent with those of their local clouds. Using the kinematic data from Tobin et al. (2009), Alves \& Bouy (2012) show that the velocity dispersion of young stars near NGC 1980 is much smaller than those in other parts of Orion, though their kinematics are consistent with the gas. Therefore, they concluded that NGC 1980 is a distinct population from others. In this work, we use a better data set to revisit this issue. Our data are collected from the SDSS APOGEE INfrared Spectroscopy of Young Nebulous Clusters program (IN-SYNC) survey of the Orion A molecular cloud (see a detailed description in $\mathrm{Da}$ Rio et al. 2016). In the region studied here, we find more than 2200 sources, which have been observed with SDSS and have velocity uncertainties of less than $0.5 \mathrm{~km} \mathrm{~s}^{-1}$. The size of this sample is much larger than the one (287 sources) with the same accuracy from Tobin et al. (2009). Among the APOGEE sample, more than 1650 sources can be classified as young stars from the X-ray emission or infrared excess emission. In this data set, we find 280 sources in the foreground population. In Figure 19 (left), we show their radial velocity data as well as those of the other young stars in Orion A. As a comparison, we also show the PV diagrams for the gas emission in the Orion A cloud. We do not find any significant difference between the kinematics of the foreground population, other young stars, and the gas material in Orion, which confirms the previous result in Tobin et al. (2009). Futhermore, we derive the velocity dispersions of all the young stars in Figure 19 (left) as was done in Tobin et al. (2009). However, we do not find significant difference between the the velocity dispersions of young stars at the different locations of Orion A. Therefore, our study suggests that the foreground population, as other young stars in this region, are associated with the Orion A cloud. Here, we must stress that the kinematic study is only based on the radial velocities of the stars, and can be improved in the future with their proper motions from Gaia 


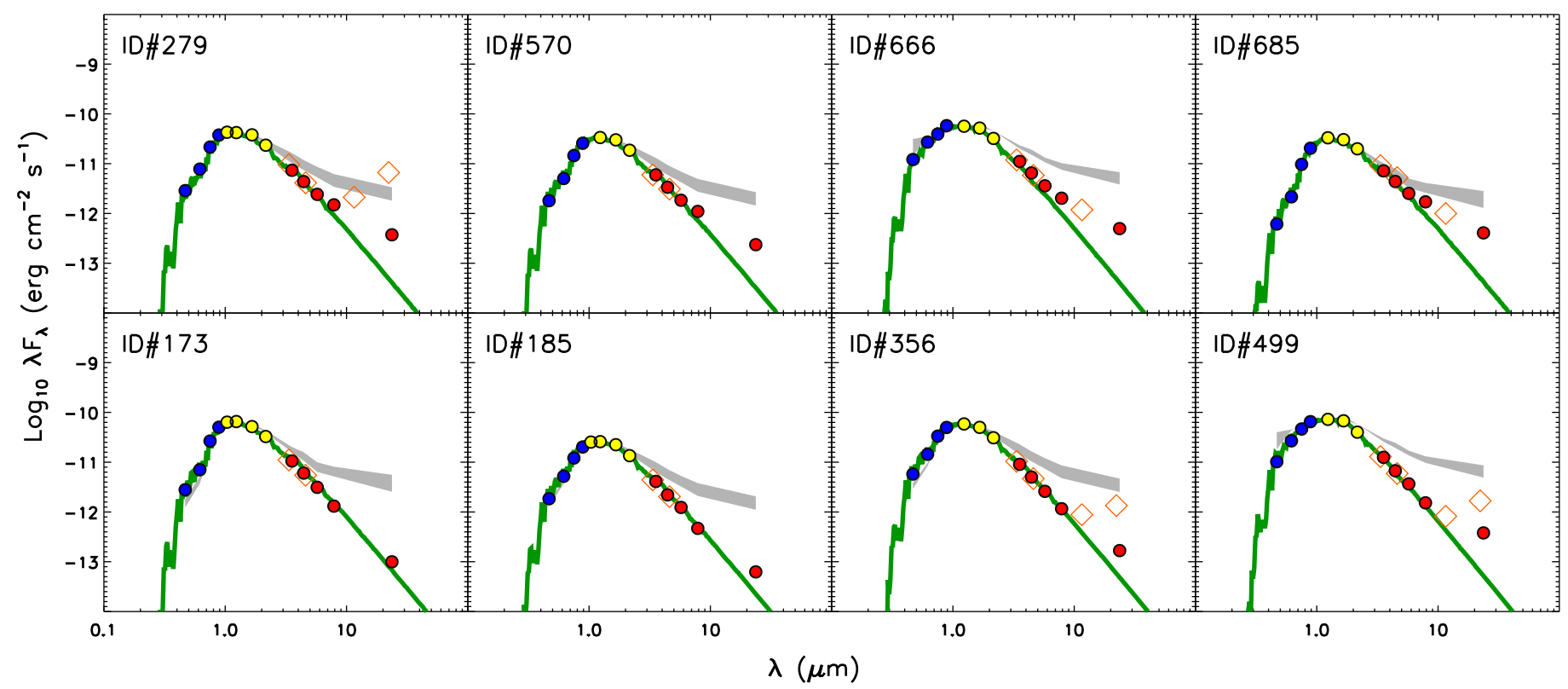

Figure 16. Same as in Figure 15, but for the SEDs of other evolved disks.

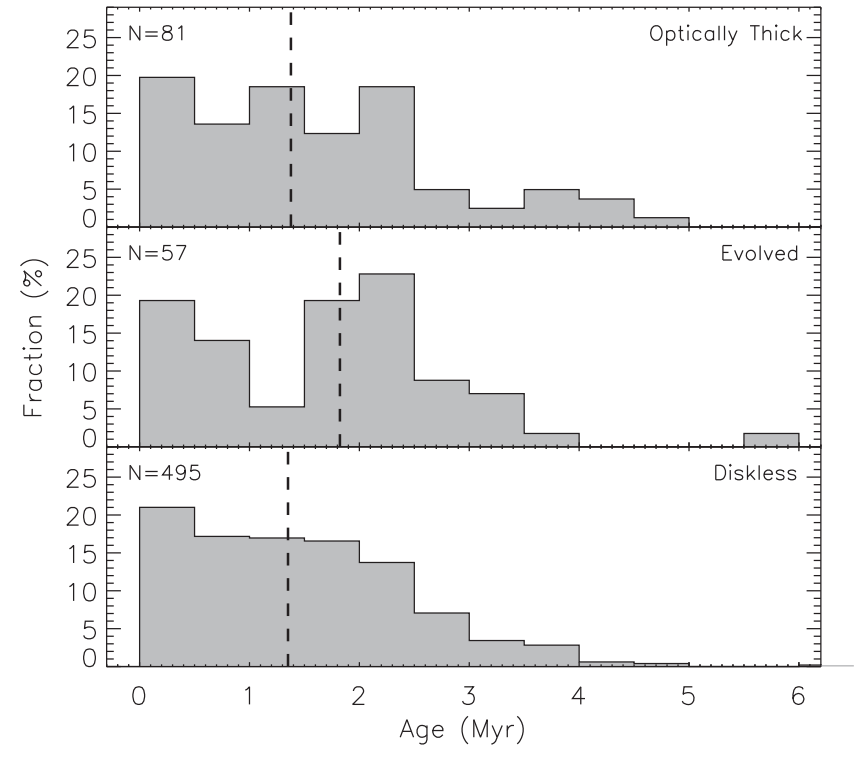

Figure 17. Histograms showing the age distribution for the young stars with optically thick disks, evolved disks, and without disks, respectively. The dashed line in each panel shows the median age of each population.

\subsection{Is the Foreground Population Older than Other Regions in Orion A?}

As shown in Figure 10 (right), the median age of the foreground population with spectroscopic data is around $1.4 \mathrm{Myr}$, which is younger than the age (4-5 Myr) proposed by Alves \& Bouy (2012) for this population. In the previous work, the age is deduced based on two arguments: (1) the age of $\iota$ Ori and (2) the median SED of the disk population in NGC 1980. Gies \& Bolton (1986) revealed that $\iota$ Ori is an eccentric binary (O9 III+B1 III), and proposed that it could be formed as a result of a binary-binary encounter that ejected the two runaways (AE Aur, $\mu \mathrm{Col}$ ), which
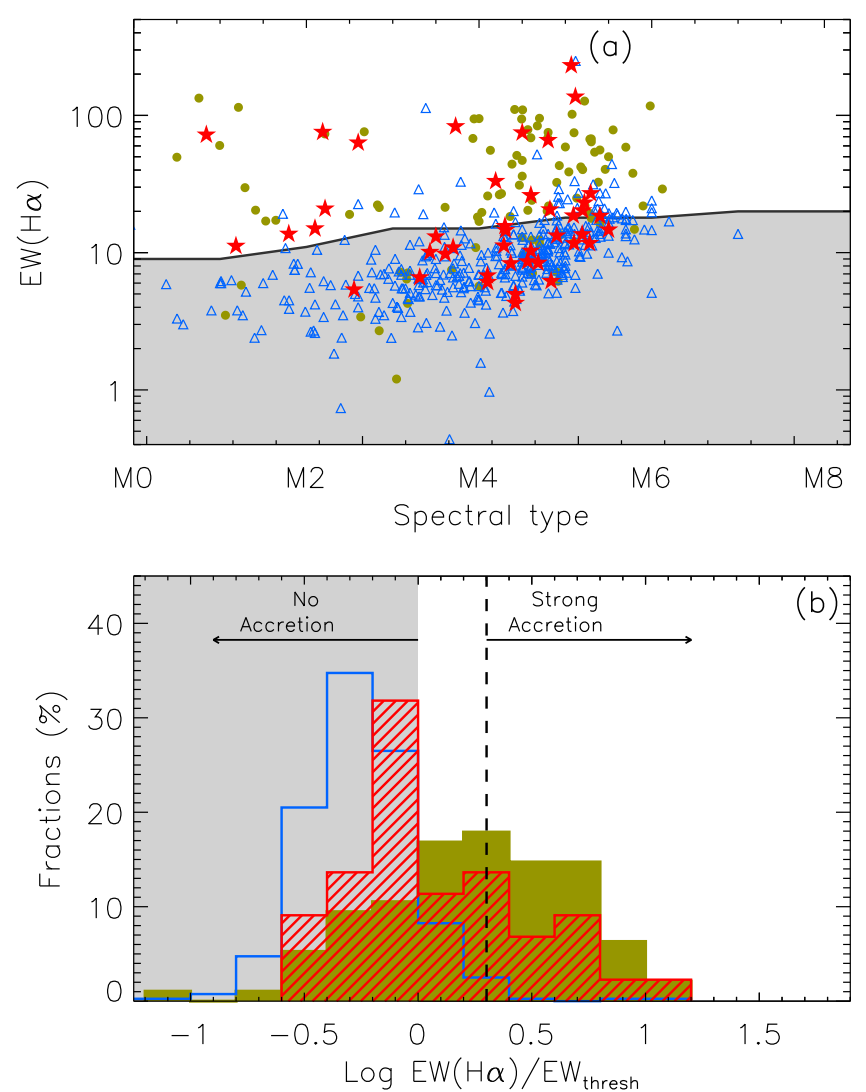

Figure 18. $\mathrm{H} \alpha$ EWs vs. spectral type for PMS stars in this work. The filled circles are for YSOs with optical thick disks $\left(\alpha_{3.6-8} \geqslant-1.8\right)$, and triangles for YSOs without disks. The star symbols show the transition disks in our sample. (b) The distribution of the ratio between the observed $\mathrm{H} \alpha \mathrm{EW}$ and the EW threshold, separating CTTSs or WTTSs, for the three populations in the left panel: YSOs with optically thick disks (filled histogram), YSOs without disks (open histogram), and transition disks (line-filled histogram). The dashed line marks $\mathrm{EW}(\mathrm{H} \alpha) / \mathrm{EW}_{\text {thresh }}=2$. 

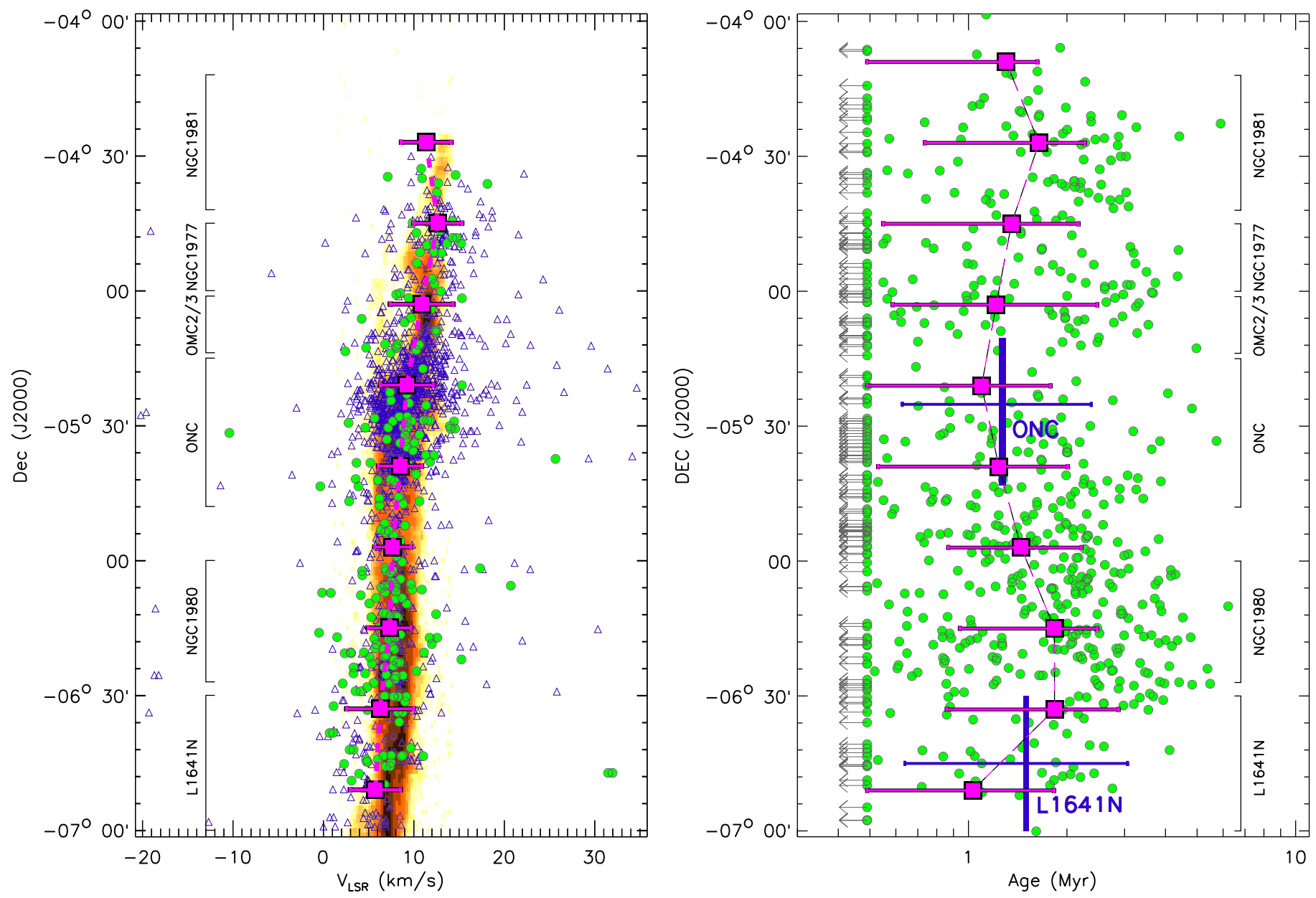

Figure 19. Left: position-velocity (PV) of both stellar radial velocities and ${ }^{13} \mathrm{CO}(J=1-0)$ emission (background color map) along the Orion A cloud. The filled circles are for the foreground population in Bouy et al. (2014), and triangles for young stars in Orion. The ${ }^{13} \mathrm{CO}$ emission is from Bally et al. (1987). The PV plot for the ${ }^{13} \mathrm{CO}$ emission is summed over the range of R.A. shown in Figure 1. The filled squares connected with the dashed line show the mean radial velocities for individual declination bins with the err-bars showing the standard deviations. Right: the ages of the young stars in this work vs. their Declination. The left pointing arrow indicates the up-limits of ages for the stars with ages above the youngest isochrone ( 0.5 Myr) in the PMS evolutionary models of Baraffe et al. (2015). The filled squares connected with the dashed line show the median ages for individual declination bins, and the err-bars show age ranges including $50 \%$ of the sources near the median ages. The median ages of ONC and L1641 are also shown in the figure as a comparison.

is confirmed by Hoogerwerf et al. (2000) using the Hipparcos data supplemented with the available radial velocities. The trapezium cluster is the most likely parent for the three sources. Therefore, it is still unclear if the $\iota$ Ori is related to the foreground population, and therefore uncertain to use the age of $\iota$ Ori as the one of the whole foreground population. When comparing the shapes of SEDs, Alves \& Bouy (2012) normalized the SEDs of different regions to the $J$-band flux. We note that there are systematic differences between the SEDs of these regions at wavelengths shorter than the $J$ band, which may suggest that the extinctions of different regions have not been corrected when they constructed the SEDs. We estimate how the extinction affects the spectral slopes. For $A_{\mathrm{V}}=5$, the dereddened spectral slope between the $J$ band and the Spitzer $8 \mu \mathrm{m}$ band can be 0.5 less than the observed spectral slopes. In Figure 20, we compare the median SED of class II sources in this work with those in the Orion nebula cluster (ONC, 1 Myr), L1641 (1.5 Myr), and $\sigma$ Ori (3 Myr), using the data from the literature (Hillenbrand 1997; Da Rio et al. 2010; Megeath et al. 2012; Fang et al. 2009, 2013a; Hernández et al. 2007b, 2014). These regions have been extensively surveyed with spectroscopy, which provides a reliable estimate of extinction for each source. In this work, most of the sources are mid-M spectral types. Therefore, we only include the M-type stars for constructing the median SEDs. We divide the sources into two groups according to their spectral types: M2-M4 and M4-M6, since the luminosity of central stars can affect the infrared spectral slopes. For individual sources, their SEDs are first extinction-corrected before combination. The extinction law is from Schlafly \& Finkbeiner (2011) for the SDSS bands adopting a total to selective extinction value typical of interstellar medium dust $\left(R_{\mathrm{V}}=3.1\right)$, Rieke \& Lebofsky (1985) for the 2MASS bands, and Flaherty et al. (2007) for the Spitzer bands. Figure 20 shows the median SEDs of different clusters. After correcting the extinction, we do not see any significant difference between the foreground population and other regions in Orion, especially for the M4-M6 group. The low disk fraction (28\%) of this population may indicate that this population may be older than its median isochronal age according to the relation between disk fractions and ages (Haisch et al. 2001; SiciliaAguilar et al. 2006; Hernández et al. 2007b; Fang et al. 2012, 2013b). However, this population is most likely biased against sources with hot inner disks, those showing $K_{\mathrm{s}}$-band excess emission, since their sample selection criteria select stars with $i-K_{\mathrm{S}}$ or $H-K_{\mathrm{s}}$ colors similar to the intrinsic photospheric colors (Alves \& Bouy 2012; Bouy et al. 2014). The typical fraction of sources with hot inner disks is $\sim 40 \%-50 \%$ for a young 


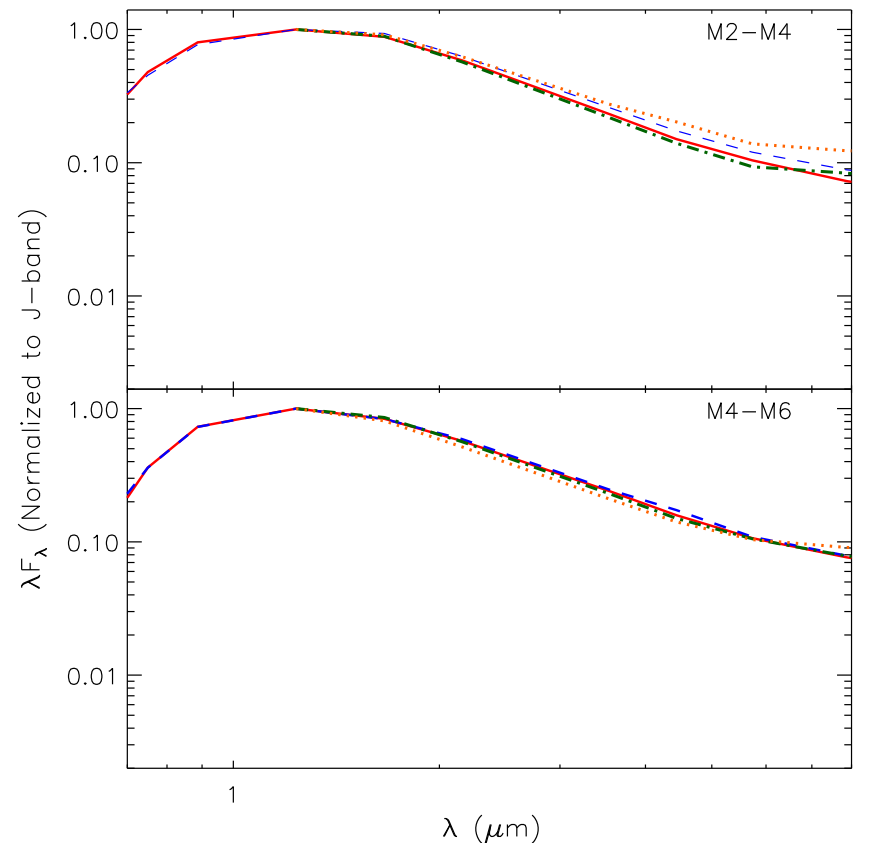

Figure 20. Median SEDs of class II sources with spectral types M2-M4 (upper) and M4-M6 (lower) in clusters of ONC (dotted line, 1 Myr), L1641 (dash line, 1.5 Myr), NGC 1980 (solid line), and $\sigma$ Ori (dashed-dotted line, 3 Myr).

population with an age of 1-2 Myr (Hillenbrand 2005; Yasui et al. 2010). The low disk fraction (28\%) of our sample is most likely due to the exclusion of sources with hot inner disks.

In Figure 19 (right), we show the ages of sources in the foreground population versus their declination. In different declinations, the ages of the stars all show large age spread with ages ranging from $\lesssim 0.5 \mathrm{Myr}$ to $\sim 6 \mathrm{Myr}$. We divide the sources into different groups according to their declination, and derive the median ages. In the figure, we show these median ages as well as the bars, which show the ranges including $50 \%$ of sources near the median ages. The median ages of the foreground population in the whole region are all around 1-2 Myr and are clearly younger than the age (4-5 Myr) proposed in Alves \& Bouy (2012). Along the declination, though it is not statistically significant, the median ages of different regions seem to show a slight gradient, and the median ages of the stars around NGC 1980 are slightly older (1.8 Myr) than other regions, which is consistent with the result in Da Rio et al. (2016). As a comparison, we show the median ages of ONC (1.3 Myr) and L1641N (1.5 Myr). For both regions, we re-estimate their ages using the same PMS evolutionary tracks from Baraffe et al. (2015) as we did for the foreground population. The effective temperature and luminosity of each star in ONC is collected from Da Rio et al. (2010), and those in L1641N are from Fang et al. (2013a). In addition, we only include the sources with spectral types between K7-M6, which is in range of spectral types for the foreground population in this work. As shown in Figure 19 (right), the median ages (1-2 Myr) of the foreground population are consistent with the ones of ONC and L1641N.

\subsection{Is the Foreground Population from One Cluster?}

In Alves \& Bouy (2012) and Bouy et al. (2014), they proposed that the foreground population in Orion is from one cluster and centered on NGC 1980. However, as discussed in the above two sections, we can see that the kinematics of the foreground population are consistent with their local clouds and other young stars in the same regions. In Figure 19 (left), the gradient in the kinematics of Orion A cloud can be clearly shown in the kinematics of the foreground population, which is a strong evidence that the foreground population is associated with the local clouds instead of being one cluster. Furthermore, we may see an age gradient along the declination, and the median age of the sources near NGC 1980 is slightly higher than others. Such an age gradient should not be seen if the foreground population is from the same cluster. Therefore, our results argue against the presence of an old, large foreground cluster in front of Orion A. We propose that the foreground population seen in Alves \& Bouy (2012) and Bouy et al. (2014) is a combination of young stars with low or no extinction in NGC 1980 and other regions in Orion A.

\section{Summary}

We performed a spectroscopic survey of the foreground population in Orion A with MMT/Hectospec. We combine the Hectospec data with optical and infrared photometric data to estimate the stellar effective temperatures, luminosities, and extinction values of individual sources, and derive masses and ages of individual sources by their placement in the H-R diagram. The disk properties of individual sources are characterized using Spitzer and WISE data, and their accretion properties are characterized using the $\mathrm{H} \alpha$ line. We also use archival APOGEE radial velocity data to study the kinematics of the foreground population and other young stars in the Orion A cloud. The main results are summarized as follows.

1. We present a catalog of 691 young stars, including their spectral types, line-of-sight extinction, stellar masses and ages, disk properties, and accretion properties.

2. We find one new subluminous object in our spectroscopic sample, and explain it as a star with a nearly edgeon disk.

3. We discovered an object with extremely low mass $\left(<0.018-0.030 M_{\odot}\right)$. The SED modeling indicates this object possesses a flaring disk.

4. We identify 37 new TD objects, 1 globally depleted disk candidate, and 7 young debris disk candidates. We investigate the accretion properties of YSOs with disks in our sample based on $\mathrm{H} \alpha$ EWs. We find that the fraction of accretors among TDs is much lower than among the YSOs with optically thick disks $(46 \% \pm 7 \%$ versus $73 \% \pm 9 \%$, respectively), which confirms our previous results in L1641.

5. We confirm that the kinematics of the foreground population is consistent with their local clouds and other young stars in the same regions in Orion A. The median age of the foreground population is also similar to those of other young stars in Orion A. Therefore, our results suggest that the foreground population is associated with Orion $\mathrm{A}$, and prove that it is not a distinct large and old (4-5 Myr) cluster in front of Orion A.

Many thanks to the anonymous referee for comments that helped to improve this paper. This material is based upon work supported by the National Aeronautics and Space Administration under Agreement No. NNX15AD94G for the program "Earths in Other Solar Systems." The results reported herein 
Table 4

The Parameters for Young Stars with X-Shooter Spectra

\begin{tabular}{|c|c|c|c|c|c|c|c|c|c|c|c|c|}
\hline ID & Name & $\begin{array}{l}\text { R.A. } \\
\text { (J2000) }\end{array}$ & $\begin{array}{c}\text { Decl. } \\
\text { (J2000) }\end{array}$ & Spt & $\operatorname{Ref}^{a}$ & $\begin{array}{l}\text { Adopted } \\
\text { Spt }\end{array}$ & $\operatorname{Ref}^{b}$ & $\begin{array}{c}\mathrm{EW}_{\mathrm{H} \alpha} \\
(\AA)\end{array}$ & $L_{\text {acc }} / L_{\star}$ & Accreting & $\begin{array}{c}A_{\mathrm{V}} \\
(\mathrm{mag})\end{array}$ & $\operatorname{Ref}^{c}$ \\
\hline 1 & RECX 1 & 083656.24 & -785645.7 & K4, K5, K6, K7 & $1,2,3,8$ & K5 & 3 & -1.2 & $\ldots$ & $\mathrm{N}$ & 0 & $\cdots$ \\
\hline 2 & RECX 5 & 084227.088 & -785747.93 & M3.8, M4 & $2,3,8$ & M4 & 3 & -12.9 & $\ldots$ & $\mathrm{N}$ & 0 & $\ldots$ \\
\hline 3 & RECX 6 & 084238.770 & -785442.75 & M2, M3 & $2,3,8,9$ & M3 & 3 & -4.8 & $\ldots$ & $\mathrm{N}$ & 0 & $\ldots$ \\
\hline 4 & RECX 7 & 84307.239 & -790452.49 & K3, K4, K5, K6.9 & $2,3,8,10$ & K5 & 3 & -1.0 & & $\mathrm{~N}$ & 0 & $\ldots$ \\
\hline 5 & RECX 9 & 084416.41 & -785908.04 & M4, M4.5 & $2,3,8$ & M4.5 & 3 & -12.4 & $\ldots$ & $\mathrm{N}$ & 0 & $\ldots$ \\
\hline 6 & RECX 10 & 084431.90 & -784631.2 & K7, K9, M0.3, M1 & $2,3,8,9$ & K9 & 3 & -1.7 & $\ldots$ & $\mathrm{N}$ & 0 & $\ldots$ \\
\hline 7 & RECX 11 & 084701.63 & -785934.37 & K5, K5.5, K6.5 & $2,3,8$ & K5 & 3 & -8.9 & 0.007 & $\mathrm{Y}$ & 0 & 28 \\
\hline 8 & RECX 12 & 084756.766 & -785453.19 & M2, M3.2, M3.25 & $2,3,8,9$ & M3.25 & 3 & -8.1 & $\ldots$ & $\mathrm{N}$ & 0 & $\ldots$ \\
\hline 9 & RECX 17 & 083851.50 & -791613.7 & M5, M5.25, M5.75 & $2,3,8$ & M5.25 & 3 & -12.1 & $\ldots$ & $\mathrm{N}$ & 0 & $\ldots$ \\
\hline 10 & RECX 18 & 083610.73 & -790818.4 & M5.5 & $2,3,8$ & M5.5 & 3 & -12.5 & & $\mathrm{~N}$ & 0 & $\ldots$ \\
\hline 11 & TWA 2A & 110913.80 & -300139.9 & $\mathrm{M} 1.5, \mathrm{M} 2$ & $3,4,5$ & M2 & 3 & -2.5 & $\ldots$ & $\mathrm{N}$ & 0 & $\ldots$ \\
\hline 12 & TWA 7 & 104230.064 & -334016.62 & M2, M3 & 3,6 & M3 & 3 & -5.2 & $\ldots$ & $\mathrm{N}$ & 0 & $\ldots$ \\
\hline 13 & TWA 8A & 113241.25 & -265155.9 & M2, M3 & $3,6,7$ & M3 & 3 & -8.8 & $\ldots$ & $\mathrm{N}$ & 0 & $\ldots$ \\
\hline 14 & TWA 8B & 113241.16 & -265209.0 & M5, M5.5 & 3,7 & M5.5 & 3 & -17.2 & $\ldots$ & $\mathrm{N}$ & 0 & $\ldots$ \\
\hline 15 & TWA 9A & 114824.223 & -372849.15 & K5, K7 & 3,7 & K7 & 3 & -1.7 & $\ldots$ & $\mathrm{N}$ & 0 & $\ldots$ \\
\hline 16 & TWA 9B & 114823.73 & -372848.5 & M1, M3.5 & 3,4 & M3.5 & 3 & -5.4 & & $\mathrm{~N}$ & 0 & $\ldots$ \\
\hline 17 & TWA 13A & 112117.24 & -344645.5 & M1 & 3,6 & M1 & 3 & -8.4 & $\ldots$ & $\mathrm{N}$ & 0 & $\ldots$ \\
\hline 18 & TWA 13B & 112117.24 & -344645.5 & M1 & 3,6 & M1 & 3 & -2.0 & $\ldots$ & $\mathrm{N}$ & 0 & $\ldots$ \\
\hline 19 & TWA 22 & 101726.89 & -535426.5 & M5 & 3 & M5 & 3 & -13.0 & $\ldots$ & $\mathrm{N}$ & 0 & $\ldots$ \\
\hline 20 & TWA 25 & 121530.72 & -394842.6 & K9, M0 & 3,4 & K9 & 3 & -3.0 & $\ldots$ & $\mathrm{N}$ & 0.4 & $\ldots$ \\
\hline 21 & TWA 26 & 113951.140 & -315921.50 & M8 & 3,6 & M8 & 3 & -12.3 & $\ldots$ & $\mathrm{N}$ & 0 & $\ldots$ \\
\hline 22 & TWA 27 & 120733.467 & -393254.00 & M8 & 3,6 & M8 & 3 & -194.4 & 0.003 & $\mathrm{Y}$ & 0 & 43 \\
\hline 23 & TWA 28 & 110209.833 & -343035.53 & M8.5 & 3,13 & M8.5 & 3 & -101.4 & 0.0093 & $\mathrm{Y}$ & 0 & 43 \\
\hline 24 & TWA 29 & 124514.16 & -442907.7 & M9.5 & $3,4,12$ & M9.5 & 3 & -10.6 & $\ldots$ & $\mathrm{N}$ & 0 & $\ldots$ \\
\hline 25 & TWA 30A & 113218.314 & -301951.85 & M5 & 3,18 & M5 & 3 & -6.6 & $\ldots$ & $\mathrm{N}$ & 0 & $\ldots$ \\
\hline 26 & V4046sgr & 181410.466 & -324734.50 & K4, K5 & 3,17 & K4 & 3 & -44.0 & 0.096 & $\mathrm{Y}$ & 0 & 42 \\
\hline 27 & RECX 4 & 084223.77 & -790403.0 & M0, M1.3, M1.75 & $2,3,8$ & M1.3 & 2 & -4.2 & $\ldots$ & $\mathrm{N}$ & 0 & $\ldots$ \\
\hline 28 & TWA 3A & 111027.81 & -373153.2 & M3, M4 & 5,6 & M4 & 5 & -9.8 & & $\mathrm{~N}$ & 0 & $\ldots$ \\
\hline 29 & TWA 3B & 111027.88 & -373152.0 & M3.5, M4 & 5,6 & M4 & 5 & -6.2 & $\ldots$ & $\mathrm{N}$ & 0 & $\ldots$ \\
\hline 30 & TWA 6 & 101828.700 & -315002.85 & K7, M0 & 4,11 & K7 & 11 & -4.5 & $\ldots$ & $\mathrm{N}$ & 0 & $\ldots$ \\
\hline 31 & TWA 14 & 111326.221 & -452342.74 & M0, M0.5 & 4,11 & M0 & 11 & -6.1 & $\ldots$ & $\mathrm{N}$ & 0 & $\ldots$ \\
\hline 32 & TWA $15 \mathrm{~A}$ & 123420.65 & $-48 \quad 1513.50$ & M2 & 6 & M3 & this work & -12.8 & $\ldots$ & $\mathrm{N}$ & 0 & $\ldots$ \\
\hline 33 & TWA 15B & 123420.47 & $-48 \quad 1519.50$ & M2 & 6 & M3 & this work & -9.5 & $\ldots$ & $\mathrm{N}$ & 0 & $\ldots$ \\
\hline 34 & Sz 74 & 154805.228 & -351552.83 & M1.5, M3.5 & 14,15 & M3.5 & 14 & -22.8 & 0.030 & $\mathrm{Y}$ & 1.5 & 14 \\
\hline 35 & Sz 84 & 155802.53 & -373602.7 & M5, M5.5 & 14,15 & M4.5 & this work & -113.2 & 0.021 & $\mathrm{Y}$ & 0.8 & 25 \\
\hline 36 & Sz 91 & 160711.592 & -390347.54 & M0.5, M1, M1.5 & $14,15,16$ & M1 & 14 & -139.6 & 0.051 & $\mathrm{Y}$ & 1.2 & 14 \\
\hline 37 & Sz 94 & 160749.596 & -390428.79 & M4 & 4,15 & M3.5 & this work & -6.4 & $\ldots$ & $\mathrm{N}$ & 0.0 & $\ldots$ \\
\hline 38 & Sz 97 & 160821.803 & -390421.48 & M3, M4 & 14,15 & M4 & 14 & -38.6 & 0.007 & $\mathrm{Y}$ & 0 & 14 \\
\hline 39 & Sz 100 & 160825.764 & -390601.19 & M5, M5.5 & 14,15 & M5.5 & 14 & -45.1 & 0.006 & $\mathrm{Y}$ & 0 & 14 \\
\hline 40 & Sz 104 & 160830.815 & -390548.87 & M5, & 14,15 & M5 & 14 & -34.1 & 0.006 & $\mathrm{Y}$ & 0 & 14 \\
\hline 41 & Sz 107 & 160841.799 & -390137.02 & M5.5, M5.75 & 4,19 & M5.5 & 4 & -12.8 & & $\mathrm{~N}$ & 0 & $\ldots$ \\
\hline 42 & Sz 111 & 160854.687 & -393743.11 & M1, M1.5 & 14,15 & M0 & this work & -79.7 & 0.019 & $\mathrm{Y}$ & 0.7 & 14 \\
\hline 43 & Sz 112 & 160855.530 & -390233.95 & M4, M5, M6 & $14,15,19$ & M5 & 14 & -17.4 & $\ldots$ & $\mathrm{N}$ & 0 & $\ldots$ \\
\hline 44 & Sz 114 & 160901.850 & -390512.42 & M4, M4.8, M5.5 & $14,15,20$ & M4.8 & 14 & -94.4 & 0.010 & $\mathrm{Y}$ & 0 & 14 \\
\hline 45 & Sz 115 & 160906.214 & -390851.86 & M4, M4.5 & 14,15 & M4.5 & 14 & -10.9 & $\ldots$ & $\mathrm{N}$ & 0 & $\ldots$ \\
\hline 46 & Sz 121 & 161012.199 & -392118.11 & M3, M4 & $4,15,24$ & M4 & 24 & -7.8 & $\ldots$ & $\mathrm{N}$ & 0 & $\ldots$ \\
\hline 47 & Sz 122 & 161016.424 & -390805.07 & M2 & $4,15,24$ & M2 & 4 & -6.9 & $\ldots$ & $\mathrm{N}$ & 0 & $\ldots$ \\
\hline 48 & SO 587 & 053834.04 & -023637.3 & M3.5, M4.5 & 21,22 & M4.5 & 22 & -16.3 & $\ldots$ & $\mathrm{N}$ & 0 & $\ldots$ \\
\hline 49 & SO 641 & 053838.57 & -024155.8 & M5 & 4,22 & M5 & 22 & -8.7 & $\ldots$ & $\mathrm{N}$ & 0 & $\ldots$ \\
\hline 50 & SO 797 & 053854.91 & -022858.19 & M4, M4.5 & 4,22 & M4.5 & 22 & -7.7 & $\ldots$ & $\mathrm{N}$ & 0 & $\ldots$ \\
\hline 51 & SO 879 & 053905.42 & -023230.34 & K5, K7 & $4,21,24$ & K7 & 24 & -2.2 & $\ldots$ & $\mathrm{N}$ & 0 & $\ldots$ \\
\hline 52 & SO 925 & 053911.41 & -023332.8 & M5.5 & 4,22 & M5.5 & 22 & -9.6 & $\ldots$ & $\mathrm{N}$ & 0 & $\ldots$ \\
\hline 53 & SO 999 & 053920.25 & $-02: 3825.8$ & M5.5 & 4,22 & M5.5 & 22 & -11.0 & $\ldots$ & $\mathrm{N}$ & 0 & $\ldots$ \\
\hline
\end{tabular}


Table 4

(Continued)

\begin{tabular}{|c|c|c|c|c|c|c|c|c|c|c|c|c|}
\hline ID & Name & $\begin{array}{l}\text { R.A. } \\
\text { (J2000) }\end{array}$ & $\begin{array}{l}\text { Decl. } \\
\text { (J2000) }\end{array}$ & Spt & $\operatorname{Ref}^{a}$ & $\begin{array}{l}\text { Adopted } \\
\text { Spt }\end{array}$ & $\operatorname{Ref}^{b}$ & $\begin{array}{c}\mathrm{EW}_{\mathrm{H} \alpha} \\
(\AA)\end{array}$ & $L_{\text {acc }} / L_{\star}$ & Accreting & $\begin{array}{c}A_{\mathrm{V}} \\
\text { (mag) }\end{array}$ & $\operatorname{Ref}^{\mathrm{c}}$ \\
\hline 54 & Par-Lup3-1 & 160816.03 & -390304.29 & M6.5, M7.5 & 23,24 & M6.5 & 24 & -17.6 & $\ldots$ & $\mathrm{N}$ & 0 & $\ldots$ \\
\hline 55 & Par-Lup3-2 & 160835.78 & -390347.91 & M5, M6 & 23,24 & M5 & 24 & -5.5 & $\ldots$ & $\mathrm{N}$ & 0 & $\ldots$ \\
\hline 56 & Par-Lup3-3 & 160849.40 & -390539.2 & M4, M4.5 & 14,23 & M3.5 & this work & -28.2 & $\cdots$ & $\mathrm{N}$ & 3.5 & $\ldots$ \\
\hline 57 & SST-Lup3-1 & 161159.798 & -382338.34 & M5 & 14 & M5 & 14 & -44.4 & 0.004 & $\mathrm{Y}$ & 0 & 14 \\
\hline 58 & Lup706 & 160837.30 & $\begin{array}{lll}-39 & 23 & 10.8\end{array}$ & M7.75 & 14 & M7.75 & 14 & -222.5 & 0.005 & $\mathrm{Y}$ & 0 & 14 \\
\hline 59 & Lup604s & 160800.20 & -390259.7 & M5.25 & 14 & M5.25 & 14 & -16.7 & $\ldots$ & $\mathrm{N}$ & 0 & $\ldots$ \\
\hline 60 & Lup818s & 160956.29 & -385951.7 & M6 & 14 & M6 & 14 & -52.3 & 0.003 & $\mathrm{Y}$ & 0 & 14 \\
\hline 61 & CrA75 & 190222.1 & -365540.9 & $\mathrm{~K} 2$ & 25 & $\mathrm{~K} 2$ & 25 & -1.3 & & $\mathrm{~N}$ & 0.3 & $\ldots$ \\
\hline 62 & ISO-217 & 110952.15 & -763912.8 & M6.25 & 26 & M6.25 & 26 & -125.2 & 0.007 & $\mathrm{Y}$ & 2.6 & 41 \\
\hline 63 & АKC2006-19 & 154457.90 & -342339.5 & M5 & 14 & M5 & 14 & -29.8 & 0.005 & $\mathrm{Y}$ & 0 & 14 \\
\hline 64 & 2MASSJ16085953-3856275 & 160859.53 & -385627.6 & M8.5 & 14 & M8.5 & 14 & -111.5 & 0.003 & $\mathrm{Y}$ & 0 & 14 \\
\hline 65 & SSTc2d160901.4-392512 & 160901.40 & -392511.9 & M4 & 14,19 & M3 & this work & -42.7 & 0.007 & $\mathrm{Y}$ & 0.8 & 14 \\
\hline 66 & Cha $\mathrm{H} \alpha 1$ & 110716.68 & -773553.2 & M7.5, M7.75 & 26,27 & M7.75 & 26 & -125.9 & $\ldots$ & $\mathrm{N}$ & 0 & 40 \\
\hline 67 & Ass-Cha-T-2-51 & 111224.415 & -763706.41 & $\mathrm{~K} 3.5, \mathrm{~K} 4$ & 10,26 & K3.5 & 26 & -2.9 & $\ldots$ & $\mathrm{N}$ & 0 & $\ldots$ \\
\hline 68 & LkCa 15 & 043917.796 & +222103.48 & K2, K5 & $25,29,30$ & K5 & 29 & -28.3 & 0.066 & $\mathrm{Y}$ & 0.6 & 25 \\
\hline 69 & CS Cha & 110224.912 & -773335.72 & K2, K4, K5, K6 & $6,25,26,31$ & K5 & 31 & -29.5 & 0.069 & $\mathrm{Y}$ & 0.3 & 25 \\
\hline 70 & CHXR 22E & 110713.300 & -774349.88 & M3.5, M4 & 25,26 & M4 & 25 & -6.4 & $\ldots$ & $\mathrm{N}$ & 3.4 & $\ldots$ \\
\hline 71 & Sz 18 & 110719.154 & -760304.85 & M2, M2.5 & 25,26 & M2 & 25 & -25.0 & 0.048 & $\mathrm{Y}$ & 0.6 & 25 \\
\hline 72 & Sz 27 & 110839.051 & -771604.24 & $\mathrm{~K} 7, \mathrm{~K} 8$ & $25,26,29$ & K7 & 25 & -58.7 & 0.076 & $\mathrm{Y}$ & 2.8 & 25 \\
\hline 73 & RX J1615-3255 & 161520.231 & -325505.10 & K5, K7 & $25,32,33$ & K5 & 32 & -35.6 & 0.056 & $\mathrm{Y}$ & 0 & 25 \\
\hline 74 & Oph 22 & 162245.40 & -243123 & M3 & 25,34 & M3 & 25 & -5.0 & $\ldots$ & $\mathrm{N}$ & 1.4 & \\
\hline 75 & Oph 24 & 162506.91 & -235050.3 & M0, M3 & 25,34 & M2 & this work & -5.1 & & $\mathrm{~N}$ & 0 & \\
\hline 76 & ISO-Oph196 & 162816.51 & -243657.9 & M4.5, M5.5 & 25,35 & M5 & this work & -106.8 & 0.062 & $\mathrm{Y}$ & 2.0 & 25 \\
\hline 77 & Ser 29 & 182911.50 & 002038.6 & M0, M2 & 25,36 & M2 & 25 & -13.9 & $<0.004$ & $\mathrm{Y}$ & 2.9 & 25 \\
\hline 78 & Ser 34 & 182944.11 & 003356.0 & M0, M1 & 25,36 & M1.5 & this work & -13.6 & 0.008 & $\mathrm{Y}$ & 2.3 & 25 \\
\hline 79 & RX J1842.9-3532 & 184257.95 & -353242.7 & K2 & 25,37 & K2 & 37 & -32.9 & 0.056 & $\mathrm{Y}$ & 1.7 & 25 \\
\hline 80 & RX J1852.3-3700 & $1852 \quad 17.29$ & -370011.9 & K2, K3 & 25,37 & $\mathrm{~K} 3$ & 37 & -44.8 & 0.051 & $\mathrm{Y}$ & 1.6 & 25 \\
\hline 81 & LkH $\alpha 330$ & 034548 . & 29322411.9 & $\mathrm{G} 3, \mathrm{G} 4$ & 25,38 & G4 & 25 & -15.8 & 0.024 & $\mathrm{Y}$ & 2.9 & 25 \\
\hline 82 & SR 21 & 162710.28 & -241912.7 & G4 & 25 & G4 & 25 & 1.3 & 0.017 & $\mathrm{Y}$ & 5.2 & 25 \\
\hline 83 & $\mathrm{~T} 21$ & 110615.4 & -772156.9 & G5 & $25,26,29$ & G5 & 25 & -0.5 & $\ldots$ & $\mathrm{N}$ & 3.9 & $\ldots$ \\
\hline 84 & IC348-127 & 034507.9 & 320401.8 & G4 & 25,39 & G4 & 25 & -2.8 & $\ldots$ & $\mathrm{N}$ & 6.2 & \\
\hline 85 & Cha $\mathrm{H} \alpha 9$ & 110718.608 & -773251.66 & M5.5 & 30 & M5.5 & 30 & -20.1 & $\ldots$ & $\mathrm{N}$ & 5.0 & 40 \\
\hline
\end{tabular}

${ }^{\text {a }}$ The reference for the available spectral types of individual sources.

${ }^{\mathrm{b}}$ The reference for the adopted spectral type.

c The reference for the accretion luminosity.

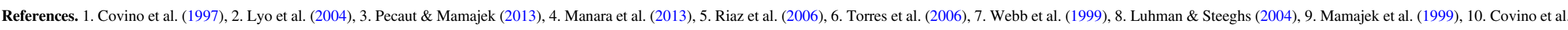

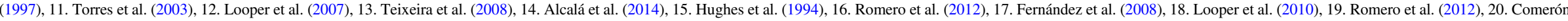

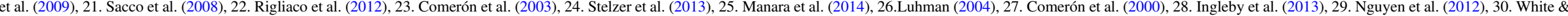

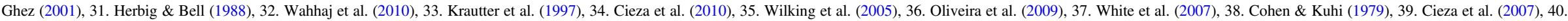
Mohanty et al. (2005), 41. Muzerolle et al. (2005), 42. Donati et al. (2011), 43. Herczeg \& Hillenbrand (2008).

(This table is available in machine-readable form.) 


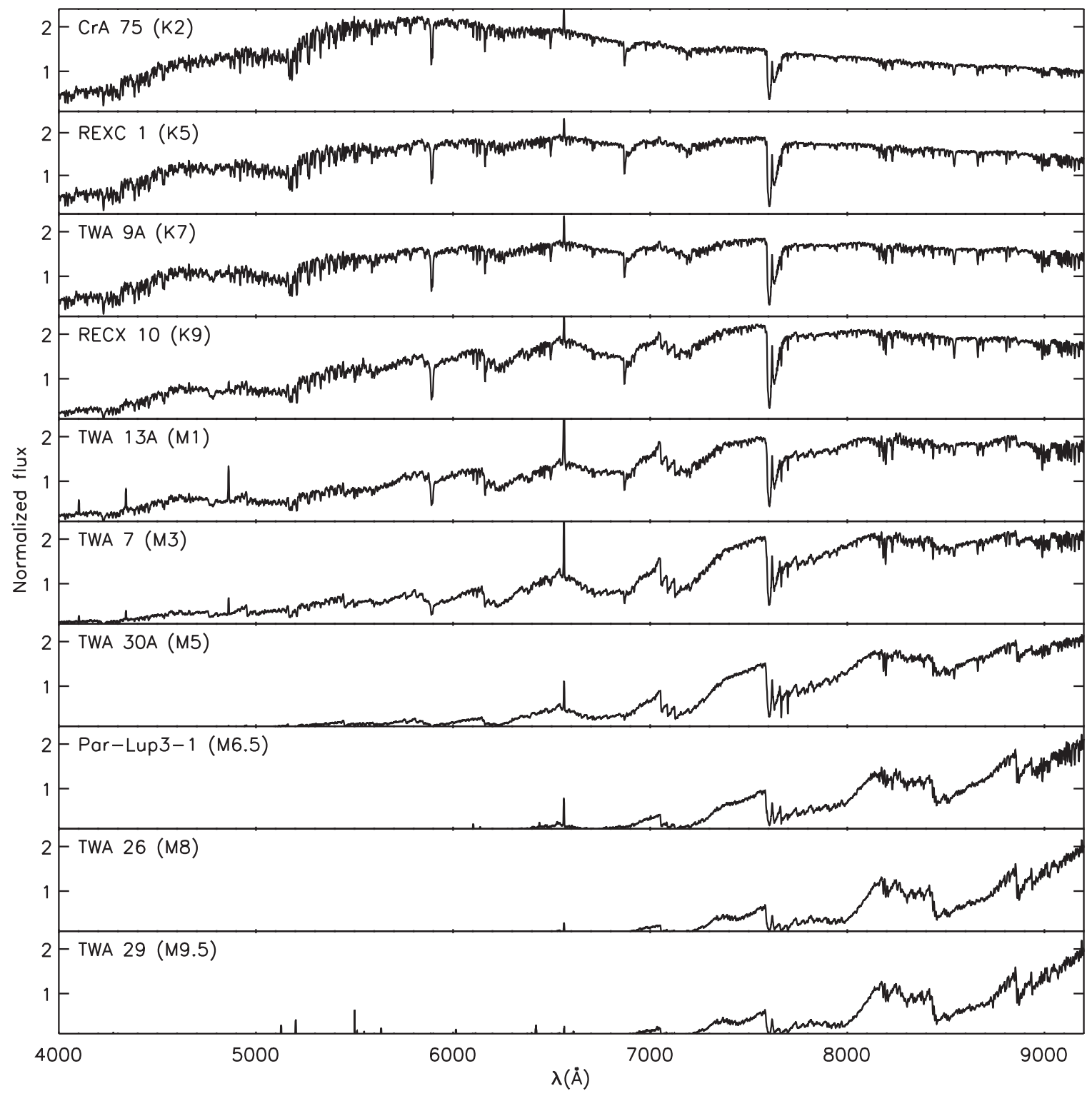

Figure 21. Examples of X-Shooter spectra, which are used to refine our spectral classification scheme.

benefitted from collaborations and/or information exchange within NASAs Nexus for Exoplanet System Science (NExSS) research coordination network sponsored by NASA's Science Mission Directorate. L.Z. acknowledges support from National Science Foundation of China (NSFC) grants 11303037 and $11390371 / 2$ and support from the Chinese Academy of Sciences (CAS) through a CAS-CONICYT Postdoctoral Fellowship administered by the CAS South America Center for Astronomy (CASSACA) in Santiago, Chile. This research uses data obtained through the Telescope Access Program (TAP), which has been funded by the National Astronomical Observatories of China, the Chinese Academy of Sciences (the Strategic Priority Research Program "The Emergence of Cosmological Structures" Grant No. XDB09000000), and the Special Fund for Astronomy from the Ministry of Finance. Observations reported here were obtained at the MMT Observatory, a joint facility of the University of Arizona and the Smithsonian Institution.

\section{Appendix A \\ Spectral Classification of Young Stars}

In Table 4, we list the young stars with X-Shooter spectra. These sources are mainly from the $\eta$ Cha cluster, the TW Hydra Association, the Lupus star-forming region, the $\sigma$ Ori cluster, and the Cha I star-forming region. We extract the spectra of these sources from the X-Shooter phase III data archive. In Figure 21, we show the example of spectra in our sample with spectral types from K2 to M9.5. In the figure, there is an obvious variation in the spectral type, which is mainly due to a change in the strength of molecular lines, including the $\mathrm{TiO}, \mathrm{VO}$, and $\mathrm{CaH}$ bands. Thus these features can be used to perform spectral classification.

We use the spectral features and define the indices of individual features in a similar way as in the spectral classification code "SPTCLASS" (Hernández et al. 2004). The index of each spectral feature is calculated by defining the central wavelengths of the feature band (FB), the blue 

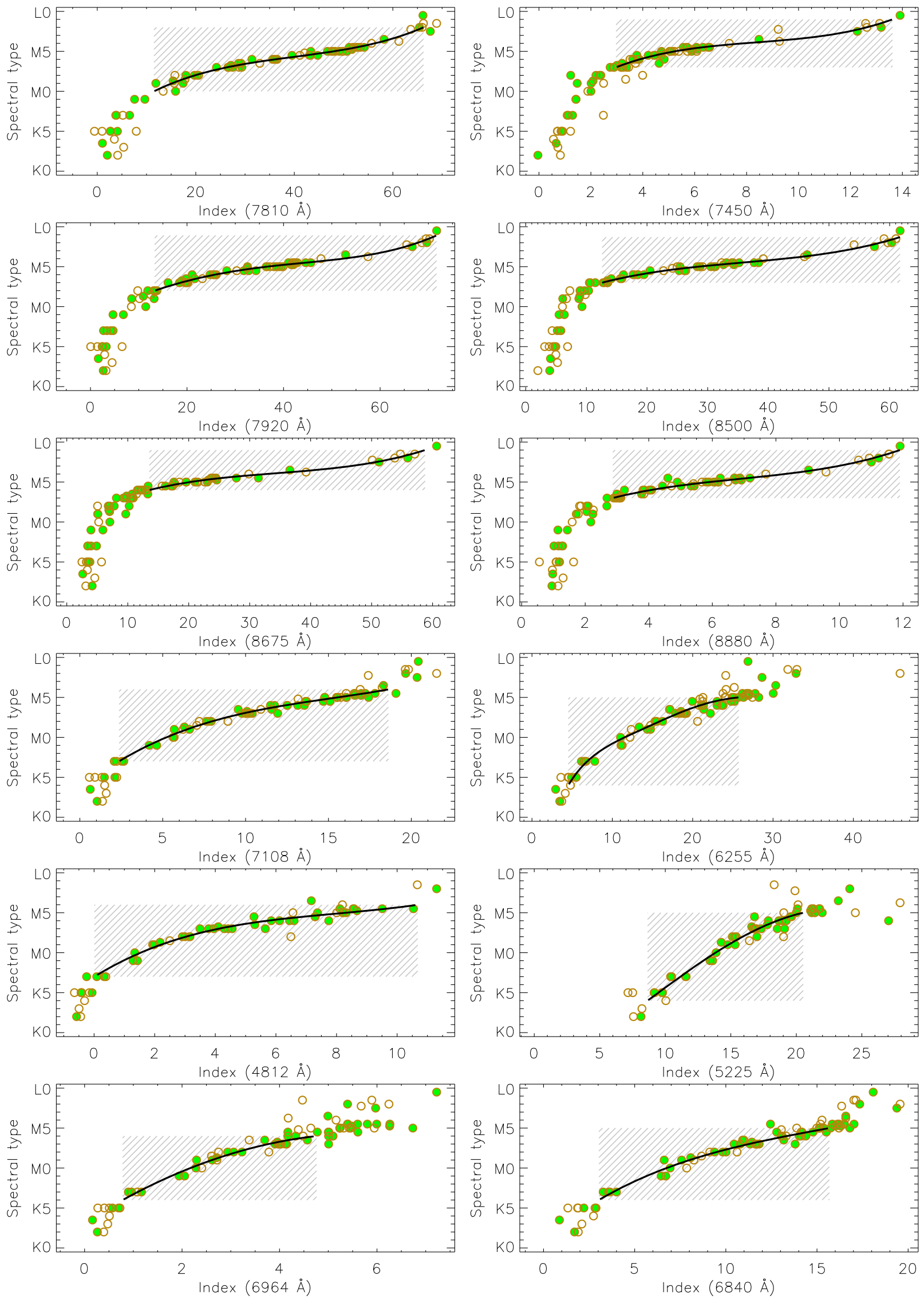

Figure 22. Relations between indices of individual spectral features and their spectral types for the young stars listed in Table 4 . The filled circles show the sources without accretion activity, and the open circles show the ones with weak accretion activity. The solid lines show the fits to the relations. The line-shaded regions mark the ranges for the fitting. 

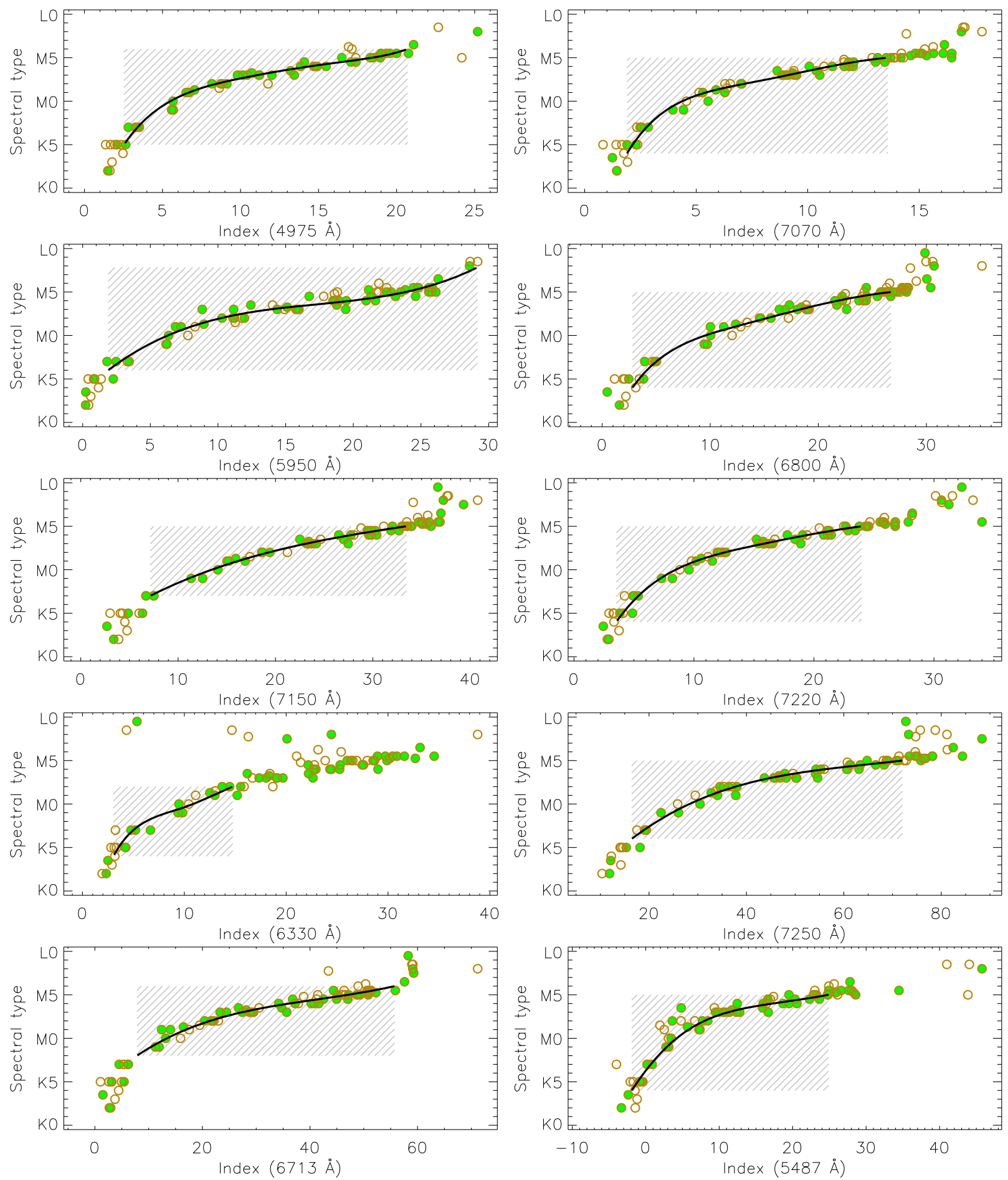

Figure 23. Same as in Figure 22, but for different spectral features.

continuum band $(\mathrm{BCB})$, and the red continuum band (RCB). The continuum flux $\left(F_{\mathrm{FCB}}\right)$ at the central wavelengths $\left(\lambda_{\mathrm{FB}}\right)$ of an FB is calculated as

$$
F_{\mathrm{FCB}}=F_{\mathrm{BCB}}+\frac{\lambda_{\mathrm{FB}}-\lambda_{\mathrm{BCB}}}{\lambda_{\mathrm{RCB}}-\lambda_{\mathrm{BCB}}} \times\left(F_{\mathrm{RCB}}-F_{\mathrm{BCB}}\right),
$$

where $\lambda_{\mathrm{BCB}}$ and $\lambda_{\mathrm{RCB}}$ are the central wavelengths of $\mathrm{BCB}$ and RCB, respectively, and $F_{\mathrm{BCB}}$ and $F_{\mathrm{RCB}}$ are the flux of BCB and RCB, respectively. $F_{\mathrm{BCB}}$ and $F_{\mathrm{RCB}}$ are the average fluxes over the widths of $\mathrm{BCB}$ and RCB. The index of an FB is calculated as

$$
\operatorname{Index}(\mathrm{FB})=\Delta \lambda_{\mathrm{FB}} \times\left(1-F_{\mathrm{FB}} / F_{\mathrm{FCB}}\right),
$$

where $\Delta \lambda_{\mathrm{FB}}$ is the width of the $\mathrm{FB}, F_{\mathrm{FB}}$ is the average flux over the width of the FB. We calculated the indices for 22 FBs. In Table 5, we list these features, including $\lambda_{\mathrm{FB}}, \Delta \lambda_{\mathrm{FB}}, \lambda_{\mathrm{BCB}}$, $\lambda_{\mathrm{RCB}}$, and the widths of the BCBs and RCBs. In Figures 22 and 23, we show the relations between the indices of individual spectral features and the spectral types. We fit these relations using polynomial functions, $\mathrm{Spt}=C_{0}+C_{1} \times$ Index 
Table 5

Optical Feature for Spectral Typing

\begin{tabular}{|c|c|c|c|c|c|c|c|c|c|c|c|c|c|c|c|}
\hline Index & Feature & $\begin{array}{c}\text { Center } \\
(\AA)\end{array}$ & $\begin{array}{l}\text { Width } \\
(\AA)\end{array}$ & $\begin{array}{l}C_{\text {left }} \\
(\AA)\end{array}$ & $\begin{array}{l}\text { Width } \\
(\AA)\end{array}$ & $\begin{array}{c}C_{\text {right }} \\
(\AA)\end{array}$ & $\begin{array}{l}\text { Width } \\
(\AA)\end{array}$ & $\begin{array}{l}\text { Spt } \\
\text { Range }\end{array}$ & $\begin{array}{l}\text { Index } \\
\text { Range }\end{array}$ & $C_{0}$ & $C_{1}$ & $C_{2}$ & $C_{3}$ & $C_{4}$ & $C_{5}$ \\
\hline 1 & VO & 7810 & 110 & 7570 & 25 & 8140 & 20 & M0-M8 & 11.51 to 66.23 & 5.44607 & 0.507010 & $-1.06971 \times 10^{-2}$ & $8.91401 \times 10^{-5}$ & 0 & 0 \\
\hline 2 & vO & 7450 & 40 & 7540 & 20 & 7540 & 20 & M3-M9.5 & 2.97 to 13.62 & 7.40348 & 2.63063 & -0.284250 & $1.12802 \times 10^{-2}$ & 0 & 0 \\
\hline 3 & VO & 7920 & 110 & 7562 & 20 & 8130 & 20 & M2-M9.5 & 13.31 to 71.70 & 8.56015 & 0.310141 & $-3.77074 \times 10^{-3}$ & $-1.51972 \times 10^{-5}$ & $4.95808 \times 10^{-7}$ & 0 \\
\hline 4 & VO & 8500 & 110 & 8408 & 10 & 8840 & 10 & M3-M9 & 12.60 to 61.72 & 9.46791 & 0.377978 & $-8.78991 \times 10^{-3}$ & $8.24764 \times 10^{-5}$ & 0 & 0 \\
\hline 5 & VO & 8675 & 150 & 8408 & 12 & 8840 & 10 & M4-M9.5 & 13.54 to 58.77 & 10.0762 & 0.410874 & $-1.03032 \times 10^{-2}$ & $1.00317 \times 10^{-4}$ & 0 & 0 \\
\hline 6 & VO & 8880 & 35 & 8850 & 10 & 9045 & 10 & M3-M9 & 2.87 to 11.90 & 8.62001 & 2.16112 & -0.256581 & $1.24604 \times 10^{-2}$ & 0 & 0 \\
\hline 7 & $\mathrm{TiO}$ & 7108 & 30 & 7040 & 15 & 7326 & 15 & K7-M6 & 2.37 to 18.62 & 3.61125 & 1.62902 & $-8.76069 \times 10^{-2}$ & $1.92530 \times 10^{-3}$ & 0 & 0 \\
\hline 8 & $\mathrm{TiO}$ & 6255 & 45 & 6112 & 15 & 6525 & 15 & K4-M5 & 4.52 to 25.74 & -9.61363 & 4.82202 & -0.522211 & $3.05772 \times 10^{-2}$ & $-8.68462 \times 10^{-4}$ & $9.40336 \times 10^{-6}$ \\
\hline 9 & $\mathrm{TiO}$ & 4812 & 18 & 4742 & 10 & 4938 & 15 & K7-M6 & 0.01 to 10.68 & 6.98046 & 2.31755 & -0.250489 & $1.05380 \times 10^{-2}$ & 0 & 0 \\
\hline 10 & $\mathrm{TiO}$ & 5225 & 75 & 4940 & 15 & 5415 & 15 & K4-M5 & 8.65 to 20.53 & -5.56706 & 0.852030 & $4.54304 \times 10^{-2}$ & $-1.85754 \times 10^{-3}$ & 0 & 0 \\
\hline 11 & $\mathrm{CaH}$ & 6964 & 22 & 6533 & 10 & 7032 & 10 & K6-M4 & 0.78 to 4.76 & 3.19107 & 3.86115 & -0.334198 & 0 & 0 & 0 \\
\hline 12 & $\mathrm{CaH}$ & 6840 & 30 & 6533 & 10 & 7032 & 10 & K6-M5 & 3.04 to 15.68 & 1.23510 & 1.83375 & $-9.31380 \times 10^{-2}$ & $2.05201 \times 10^{-3}$ & 0 & 0 \\
\hline 13 & $\mathrm{TiO}$ & 4975 & 40 & 4940 & 15 & 5385 & 15 & K5-M6 & 2.53 to 20.70 & -5.00123 & 5.58890 & -0.783747 & $5.93566 \times 10^{-2}$ & $-2.27083 \times 10^{-3}$ & $3.46253 \times 10^{-5}$ \\
\hline 14 & $\mathrm{TiO}$ & 7070 & 30 & 7045 & 10 & 7385 & 15 & K4-M5 & 1.90 to 13.59 & -8.50191 & 9.52190 & -1.87384 & 0.188538 & $-9.13227 \times 10^{-3}$ & $1.69261 \times 10^{-4}$ \\
\hline 15 & $\mathrm{TiO}$ & 5950 & 60 & 5810 & 20 & 6080 & 30 & K6-M8.5 & 1.88 to 29.18 & 3.54680 & 1.43835 & $-7.45602 \times 10^{-2}$ & $1.44120 \times 10^{-3}$ & 0 & 0 \\
\hline 16 & $\mathrm{TiO}$ & 6800 & 50 & 6530 & 15 & 7020 & 30 & K4-M5 & 2.80 to 26.72 & -2.75493 & 3.21562 & -0.336413 & $1.91640 \times 10^{-2}$ & $-5.29676 \times 10^{-4}$ & $5.61103 \times 10^{-6}$ \\
\hline 17 & $\mathrm{TiO}$ & 7150 & 50 & 7045 & 15 & 7400 & 20 & K7-M5 & 7.14 to 33.44 & 2.22419 & 0.788315 & $-1.79554 \times 10^{-2}$ & $1.73645 \times 10^{-4}$ & 0 & 0 \\
\hline 18 & $\mathrm{TiO}$ & 7220 & 50 & 7045 & 15 & 7315 & 15 & K4-M5 & 3.53 to 23.97 & -5.94651 & 3.86310 & -0.351318 & $1.70392 \times 10^{-2}$ & $-4.04817 \times 10^{-4}$ & $3.68664 \times 10^{-6}$ \\
\hline 19 & $\mathrm{TiO}$ & 6330 & 100 & 6080 & 15 & 6458 & 30 & K4-M2 & 3.02 to 14.76 & -6.93018 & 5.45853 & -0.741166 & $4.67135 \times 10^{-2}$ & $-1.06148 \times 10^{-3}$ & 0 \\
\hline 20 & $\mathrm{TiO}$ & 7250 & 150 & 7045 & 15 & 7531 & 50 & K6-M5 & 16.44 to 72.03 & -3.22166 & 0.716094 & $-1.03322 \times 10^{-2}$ & $5.41808 \times 10^{-5}$ & 0 & 0 \\
\hline 21 & $\mathrm{TiO}$ & 6713 & 100 & 6530 & 20 & 6940 & 30 & K8-M6 & 7.82 to 55.73 & 4.18776 & 0.573689 & $-1.17938 \times 10^{-2}$ & $9.51566 \times 10^{-5}$ & 0 & 0 \\
\hline 22 & $\mathrm{TiO}$ & 5487 & 76 & 5385 & 10 & 5385 & 10 & K4-M5 & -1.90 to 24.91 & 6.28645 & 1.09733 & $-5.51680 \times 10^{-2}$ & $7.34970 \times 10^{-4}$ & $2.72954 \times 10^{-5}$ & $-6.52506 \times 10^{-7}$ \\
\hline
\end{tabular}


Table 6

Stellar Properties for the YSOs

\begin{tabular}{|c|c|c|c|c|c|c|c|c|c|c|c|c|c|c|}
\hline & $\begin{array}{c}\text { R.A. } \\
(\mathrm{J} 2000)\end{array}$ & $\begin{array}{c}\text { Decl. } \\
(\mathrm{J} 2000)\end{array}$ & Spt & $\begin{array}{l}\text { Lum } \\
\left(L_{\odot}\right)\end{array}$ & $\begin{array}{c}\mathrm{Av} \\
(\mathrm{mag})\end{array}$ & $\begin{array}{l}\text { Mass } \\
\left(M_{\odot}\right)\end{array}$ & $\begin{array}{l}\text { Age } \\
\text { Myr }\end{array}$ & $\begin{array}{c}\mathrm{EW}_{\mathrm{Li} \lambda 6708 \AA} \\
(\AA)\end{array}$ & $\begin{array}{c}\mathrm{EW}_{\mathrm{H} \alpha} \\
(\AA)\end{array}$ & $\alpha_{3.6-8.0}$ & $\alpha_{3.6-24}$ & $\mathrm{TTS}^{\mathrm{a}}$ & Disk $^{b}$ & Criteria $^{c}$ \\
\hline 1 & 053150.98 & -070005.7 & K8.7 & 0.610 & 0.2 & 0.58 & 1.5 & 0.71 & -4.6 & $\ldots$ & $\ldots$ & $\mathrm{W}$ & $\mathrm{N}$ & $\mathrm{Li}$ \\
\hline 2 & 053211.59 & -060522.2 & M4.8 & 0.076 & 0.0 & 0.12 & 1.4 & $\ldots$ & -8.1 & $\ldots$ & $\ldots$ & W & $\mathrm{N}$ & $\mathrm{Na}$ \\
\hline 3 & 053212.63 & -061234.6 & M5.3 & 0.059 & 0.0 & 0.096 & 1.5 & $\ldots$ & -11.3 & $\ldots$ & $\ldots$ & W & $\mathrm{N}$ & $\mathrm{Na}$ \\
\hline 4 & 053214.78 & -060411.2 & M4.0 & 0.096 & 0.0 & 0.18 & 2.0 & $\ldots$ & -7.0 & $\ldots$ & $\ldots$ & W & $\mathrm{N}$ & $\mathrm{Na}$ \\
\hline 5 & 053219.82 & -054827.0 & M4.1 & 0.320 & 0.0 & 0.18 & $<0.5$ & 0.33 & -6.2 & $\ldots$ & $\ldots$ & $\mathrm{W}$ & $\mathrm{N}$ & $\mathrm{Li}, \mathrm{Na}$ \\
\hline 6 & 053229.64 & -061834.8 & M6.4 & 0.053 & 0.0 & 0.065 & $<0.5$ & $\ldots$ & -61.3 & $\ldots$ & $\ldots$ & $\mathrm{C}$ & $?$ & $\mathrm{Na}$ \\
\hline 7 & 053231.67 & -061518.5 & M4.7 & 0.063 & 0.0 & 0.13 & 2.1 & 0.32 & -6.4 & $\ldots$ & $\ldots$ & W & $\mathrm{N}$ & $\mathrm{Li}, \mathrm{Na}$ \\
\hline 8 & 053235.04 & -060537.3 & M3.6 & 0.696 & 0.0 & 0.21 & $<0.5$ & $\ldots$ & -0.1 & $\ldots$ & $\ldots$ & W & $\mathrm{N}$ & $\mathrm{Na}$ \\
\hline 9 & 053237.49 & -051241.3 & K8.2 & 0.327 & 0.1 & 0.67 & 4.8 & 0.84 & -4.6 & $\ldots$ & $\ldots$ & W & $\mathrm{Y}$ & $\mathrm{Li}, \mathrm{IRE}$ \\
\hline 10 & 053238.05 & -055148.4 & M3.0 & 0.562 & 0.0 & 0.26 & $<0.5$ & 0.54 & -2.4 & $\ldots$ & $\ldots$ & $\mathrm{W}$ & $\mathrm{N}$ & $\mathrm{Li}, \mathrm{Na}$ \\
\hline
\end{tabular}

Notes.

${ }^{\mathrm{a}} \mathrm{W}$ : weak-lined T-Tauri; C: classical T-Tauri.

b ?: it is unknown if there is a disk; DB: debris disk; N: no disk; TD: transition disk; Y: having a disk.

${ }^{\mathrm{c}}$ IRE: infrared Excess; Li: lithium absorption; Na: gravity sensitive sodium lines; X: X-ray emission.

(This table is available in its entirety in machine-readable form.)

$(\mathrm{FB})+C_{2} \times(\operatorname{Index}(\mathrm{FB}))^{2}+C_{3} \times(\operatorname{Inde}(\mathrm{FB}))^{3}+C_{4} \times(\operatorname{Index}$ $(\mathrm{FB}))^{4}+C_{5} \times(\operatorname{Index}(\mathrm{FB}))^{5}$, where $\mathrm{Spt}$ is the spectral-type number, and the numbers $0-19$ correspond to K0 to M9. The results are listed in Table 4. To fully cover the range of spectral types, we have included some sources with low accretion rates. For these sources, their spectral features at wavelengths longer than $5500 \AA$ are used for the fitting, to avoid the possible accretion-related veiling in the blue part of the optical spectra.

\section{References}

Alcalá, J. M., Natta, A., Manara, C. F., et al. 2014, A\&A, 561, A2

Allard, F., Homeier, D., \& Freytag, B. 2011, in ASP Conf. Ser. 448, 16th Cambridge Workshop on Cool Stars, Stellar Systems, and the Sun, ed. C. Johns-Krull, M. K. Browning, \& A. A. West (San Francisco, CA: ASP), 91

Allard, F., Homeier, D., \& Freytag, B. 2012, RSPTA, 370, 2765

Alves, J., \& Bouy, H. 2012, A\&A, 547, A97

Apai, D., Pascucci, I., Bouwman, J., et al. 2005, Sci, 310, 834

Asplund, M., Grevesse, N., Sauval, A. J., \& Scott, P. 2009, ARA\&A, 47, 481 Bally, J., Stark, A. A., Wilson, R. W., \& Langer, W. D. 1987, ApJL, 312, L45 Baraffe, I., Homeier, D., Allard, F., \& Chabrier, G. 2015, A\&A, 577, A42

Bouy, H., Alves, J., Bertin, E., Sarro, L. M., \& Barrado, D. 2014, A\&A, 564, A29

Briceño, C., Calvet, N., Hernández, J., et al. 2005, AJ, 129, 907

Briceño, C., Hartmann, L., Hernández, J., et al. 2007, ApJ, 661, 1119

Caffau, E., Ludwig, H.-G., Steffen, M., Freytag, B., \& Bonifacio, P. 2011, SoPh, 268, 255

Cieza, L., Padgett, D. L., Stapelfeldt, K. R., et al. 2007, ApJ, 667, 308

Cieza, L. A., Schreiber, M. R., Romero, G. A., et al. 2010, ApJ, 712, 925

Cohen, M., \& Kuhi, L. V. 1979, ApJS, 41, 743

Comerón, F., Fernández, M., Baraffe, I., Neuhäuser, R., \& Kaas, A. A. 2003, A\&A, 406, 1001

Comerón, F., Neuhäuser, R., \& Kaas, A. A. 2000, A\&A, 359, 269

Comerón, F., Spezzi, L., \& López Martí, B. 2009, A\&A, 500, 1045

Covey, K. R., Ivezić, Ž., Schlegel, D., et al. 2007, AJ, 134, 2398

Covino, E., Alcala, J. M., Allain, S., et al. 1997, A\&A, 328, 187

Currie, T., Lada, C. J., Plavchan, P., et al. 2009, ApJ, 698, 1

Da Rio, N., Robberto, M., Soderblom, D. R., et al. 2010, ApJ, 722, 1092

Da Rio, N., Tan, J. C., Covey, K. R., et al. 2016, ApJ, 818, 59

Danks, A. C., \& Dennefeld, M. 1994, PASP, 106, 382

de la Reza, R., Torres, C. A. O., Quast, G., Castilho, B. V., \& Vieira, G. L. 1989, ApJL, 343, L61

Donati, J.-F., Gregory, S. G., Montmerle, T., et al. 2011, MNRAS, 417, 1747

Downes, J. J., Briceño, C., Mateu, C., et al. 2014, MNRAS, 444, 1793
Downes, J. J., Román-Zúñiga, C., Ballesteros-Paredes, J., et al. 2015, MNRAS, 450, 3490

Dullemond, C. P., Juhasz, A., Pohl, A., et al. 2012, RADMC-3D: A multipurpose radiative transfer tool, Astrophysics Source Code Library, ascl: 1202.015

Fang, M., Kim, J. S., van Boekel, R., et al. 2013a, ApJS, 207, 5

Fang, M., van Boekel, R., Bouwman, J., et al. 2013b, A\&A, 549, A15

Fang, M., van Boekel, R., King, R. R., et al. 2012, A\&A, 539, A119

Fang, M., van Boekel, R., Wang, W., et al. 2009, A\&A, 504, 461

Feigelson, E., Townsley, L., Güdel, M., \& Stassun, K. 2007, in Protostars and Planets V, ed. B. Reipurth, D. Jewitt, \& K. Keil (Tucson, AZ: Univ. Arizona Press), 313

Feigelson, E. D., \& Montmerle, T. 1999, ARA\&A, 37, 363

Fernández, D., Figueras, F., \& Torra, J. 2008, A\&A, 480, 735

Flaherty, K. M., Pipher, J. L., Megeath, S. T., et al. 2007, ApJ, 663, 1069

Getman, K. V., Flaccomio, E., Broos, P. S., et al. 2005, ApJS, 160, 319

Gies, D. R., \& Bolton, C. T. 1986, ApJS, 61, 419

Güdel, M. 2004, A\&ARv, 12, 71

Haisch, K. E., Jr., Lada, E. A., \& Lada, C. J. 2001, ApJL, 553, L153

Henden, A. A., Templeton, M., Terrell, D., et al. 2016, yCat, 2336, 0

Herbig, G. H., \& Bell, K. R. 1988, Third Catalog of Emission-Line Stars of the Orion Population: 3 (Santa Cruz, CA: Lick Observatory)

Herczeg, G. J., \& Hillenbrand, L. A. 2008, ApJ, 681, 594

Herczeg, G. J., \& Hillenbrand, L. A. 2014, ApJ, 786, 97

Herczeg, G. J., \& Hillenbrand, L. A. 2015, ApJ, 808, 23

Hernández, J., Calvet, N., Briceño, C., et al. 2007a, ApJ, 671, 1784

Hernández, J., Calvet, N., Briceño, C., Hartmann, L., \& Berlind, P. 2004, AJ, 127,1682

Hernández, J., Calvet, N., Perez, A., et al. 2014, ApJ, 794, 36

Hernández, J., Hartmann, L., Megeath, T., et al. 2007b, ApJ, 662, 1067

Hernández, J., Morales-Calderon, M., Calvet, N., et al. 2010, ApJ, 722, 1226

Hillenbrand, L. A. 1997, AJ, 113, 1733

Hillenbrand, L. A. 2005, arXiv:astro-ph/0511083

Hillenbrand, L. A., Hoffer, A. S., \& Herczeg, G. J. 2013, AJ, 146, 85

Hoogerwerf, R., de Bruijne, J. H. J., \& de Zeeuw, P. T. 2000, ApJL, 544, L133

Hsu, W.-H., Hartmann, L., Allen, L., et al. 2012, ApJ, 752, 59

Hsu, W.-H., Hartmann, L., Allen, L., et al. 2013, ApJ, 764, 114

Hughes, J., Hartigan, P., Krautter, J., \& Kelemen, J. 1994, AJ, 108, 1071

Ingleby, L., Calvet, N., Herczeg, G., et al. 2013, ApJ, 767, 112

Kim, K. H., Watson, D. M., Manoj, P., et al. 2013, ApJ, 769, 149

Krautter, J., Wichmann, R., Schmitt, J. H. M. M., et al. 1997, A\&AS, 123, 329

Lawrence, A., Warren, S. J., Almaini, O., et al. 2007, MNRAS, 379, 1599

Lawson, W. A., Lyo, A., \& Bessell, M. S. 2009, MNRAS, 400, L29

Lee, H.-T., \& Chen, W. P. 2009, ApJ, 694, 1423

Looper, D. L., Burgasser, A. J., Kirkpatrick, J. D., \& Swift, B. J. 2007, ApJL, 669, L97

Looper, D. L., Mohanty, S., Bochanski, J. J., et al. 2010, ApJ, 714, 45

Luhman, K. L. 2004, ApJ, 602, 816

Luhman, K. L., Allen, L. E., Allen, P. R., et al. 2008, ApJ, 675, 1375

Luhman, K. L., \& Steeghs, D. 2004, ApJ, 609, 917 
Lyo, A.-R., Lawson, W. A., \& Bessell, M. S. 2004, MNRAS, 355, 363

Mamajek, E. E. 2012, ApJL, 754, L20

Mamajek, E. E., Lawson, W. A., \& Feigelson, E. D. 1999, ApJL, 516, L77

Manara, C. F., Testi, L., Natta, A., et al. 2014, arXiv:1406.1428

Manara, C. F., Testi, L., Rigliaco, E., et al. 2013, A\&A, 551, A107

Megeath, S. T., Gutermuth, R., Muzerolle, J., et al. 2012, AJ, 144, 192

Menten, K. M., Reid, M. J., Forbrich, J., \& Brunthaler, A. 2007, A\&A, 474,515

Mohanty, S., Jayawardhana, R., \& Basri, G. 2005, ApJ, 626, 498

Muzerolle, J., Luhman, K. L., Briceño, C., Hartmann, L., \& Calvet, N. 2005, ApJ, 625, 906

Nguyen, D. C., Brandeker, A., van Kerkwijk, M. H., \& Jayawardhana, R. 2012, ApJ, 745, 119

Oliveira, I., Merín, B., Pontoppidan, K. M., et al. 2009, ApJ, 691, 672

Pascucci, I., Apai, D., Luhman, K., et al. 2009, ApJ, 696, 143

Pecaut, M. J., \& Mamajek, E. E. 2013, ApJS, 208, 9

Rayner, J. T., Cushing, M. C., \& Vacca, W. D. 2009, ApJS, 185, 289

Rebull, L. M. 2001, AJ, 121, 1676

Rebull, L. M., Hillenbrand, L. A., Strom, S. E., et al. 2000, AJ, 119, 3026

Riaz, B., Gizis, J. E., \& Harvin, J. 2006, AJ, 132, 866

Rieke, G. H., \& Lebofsky, M. J. 1985, ApJ, 288, 618

Rigliaco, E., Natta, A., Testi, L., et al. 2012, A\&A, 548, A56

Robin, A. C., Reylé, C., Derrière, S., \& Picaud, S. 2003, A\&A, 409, 523

Romero, G. A., Schreiber, M. R., Cieza, L. A., et al. 2012, ApJ, 749, 79

Rosen, S. R., Webb, N. A., Watson, M. G., et al. 2015, arXiv:1504.07051

Sacco, G. G., Franciosini, E., Randich, S., \& Pallavicini, R. 2008, A\&A, 488, 167

Schlafly, E. F., \& Finkbeiner, D. P. 2011, ApJ, 737, 103

Sicilia-Aguilar, A., Fang, M., Roccatagliata, V., et al. 2015a, A\&A, 580, A82

Sicilia-Aguilar, A., Hartmann, L., Calvet, N., et al. 2006, ApJ, 638, 897
Sicilia-Aguilar, A., Henning, T., Dullemond, C. P., et al. 2011, ApJ, 742, 39

Sicilia-Aguilar, A., Henning, T., \& Hartmann, L. W. 2010, ApJ, 710, 597

Sicilia-Aguilar, A., Kim, J. S., Sobolev, A., et al. 2013, A\&A, 559, A3

Sicilia-Aguilar, A., Roccatagliata, V., Getman, K., et al. 2015b, A\&A, 573, A19

Simon, M. N., Pascucci, I., Edwards, S., et al. 2016, ApJ, 831, 169

Skrutskie, M. F., Cutri, R. M., Stiening, R., et al. 2006, AJ, 131, 1163

Stelzer, B., Frasca, A., Alcalá, J. M., et al. 2013, A\&A, 558, A141

Stutz, A. M., Tobin, J. J., Stanke, T., et al. 2013, ApJ, 767, 36

Teixeira, R., Ducourant, C., Chauvin, G., et al. 2008, A\&A, 489, 825

Tobin, J. J., Hartmann, L., Furesz, G., Mateo, M., \& Megeath, S. T. 2009, ApJ, 697, 1103

Torres, C. A. O., Quast, G. R., da Silva, L., et al. 2006, A\&A, 460, 695

Torres, G., Guenther, E. W., Marschall, L. A., et al. 2003, AJ, 125, 825

Vacca, W. D., \& Sandell, G. 2011, ApJ, 732, 8

Wahhaj, Z., Cieza, L., Koerner, D. W., et al. 2010, ApJ, 724, 835

Webb, R. A., Zuckerman, B., Platais, I., et al. 1999, ApJL, 512, L63

White, R. J., Gabor, J. M., \& Hillenbrand, L. A. 2007, AJ, 133, 2524

White, R. J., \& Ghez, A. M. 2001, ApJ, 556, 265

White, R. J., \& Hillenbrand, L. A. 2004, ApJ, 616, 998

Wilking, B. A., Meyer, M. R., Robinson, J. G., \& Greene, T. P. 2005, AJ, 130,1733

Wright, E. L., Eisenhardt, P. R. M., Mainzer, A. K., et al. 2010, AJ, 140, 1868

Yasui, C., Kobayashi, N., Tokunaga, A. T., Saito, M., \& Tokoku, C. 2010, ApJL, 723, L113

York, D. G., Adelman, J., Anderson, J. E., Jr., et al. 2000, AJ, 120, 1579 